\title{
Estimating Exposure of Terrestrial Wildlife
} to Contaminants

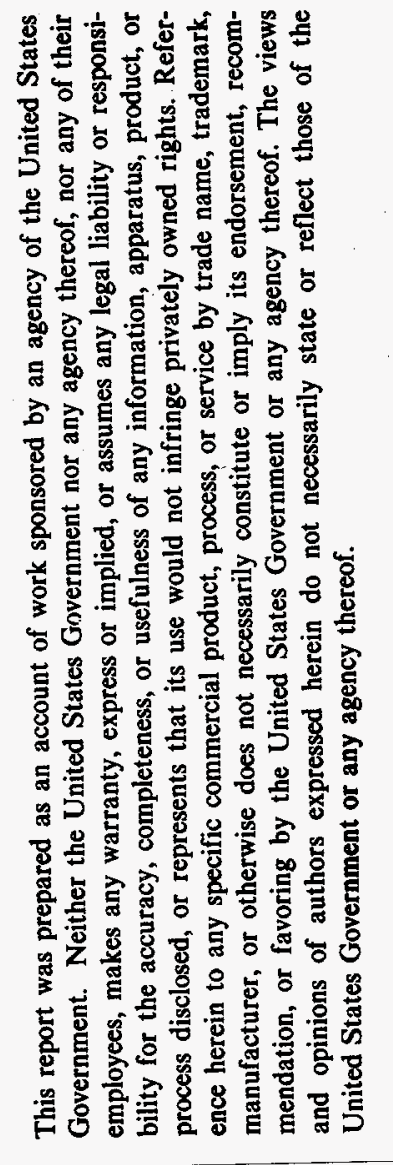

\author{
B. E. Sample \\ G. W. Suter II
}

Date Issued-September 1994

Prepared by

Environmental Sciences Division

Oak Ridge National Laboratory

Oak Ridge, Tennessee

Prepared for

U.S. Department of Energy

Office of Environmental Restoration and Waste Management under budget and reporting code EW 20

OAK RIDGE NATIONAL LABORATORY

Oak Ridge, Tennessee 37831-6285

managed by

MARTIN MARIETTA ENERGY SYSTEMS, INC.

for the

U.S. DEPARTMENT OF ENERGY

under contract DE-AC05-84OR21400 
THIS PAGE INTENTIONALLY LEFT BLANK 


\section{DISCLAIMER}

Portions of this document may be illegible in electronic image products. Images are produced from the best available original document. 


\section{CONTENTS}

ACKNOWLEDGMENTS $\ldots \ldots \ldots \ldots \ldots \ldots \ldots \ldots \ldots \ldots \ldots$

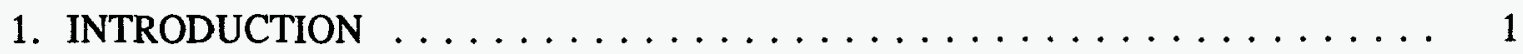

2. GENERALIZED EXPOSURE MODEL $\ldots \ldots \ldots \ldots \ldots \ldots \ldots \ldots \ldots \ldots$

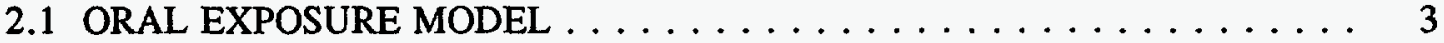

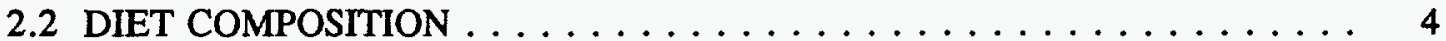

2.3 HOME RANGE/HABITAT REQUIREMENTS $\ldots \ldots \ldots \ldots \ldots \ldots \ldots$

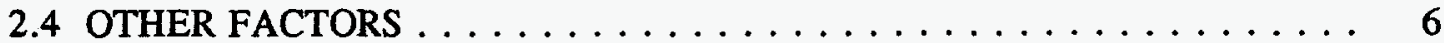

3. PARAMETERS FOR ESTIMATION OF EXPOSURE $\ldots \ldots \ldots \ldots \ldots \ldots \ldots$

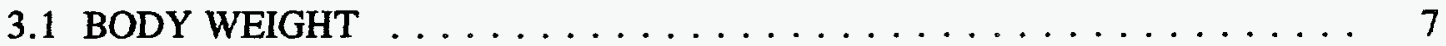

3.2 FOOD AND WATER CONSUMPTION $\ldots \ldots \ldots \ldots \ldots \ldots \ldots$

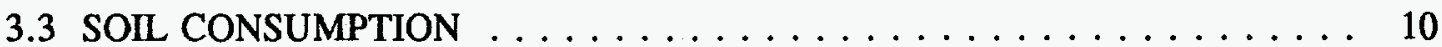

4. LIFE HISTORY PARAMETERS FOR SELECTED ENDPOINT SPECIES $\ldots \ldots \ldots$

4.1 SHORT-TAILED SHREW $\ldots \ldots \ldots \ldots \ldots \ldots \ldots \ldots \ldots \ldots \ldots$

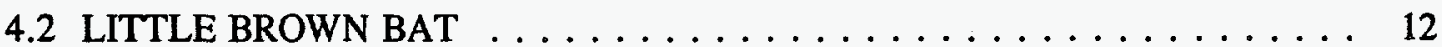

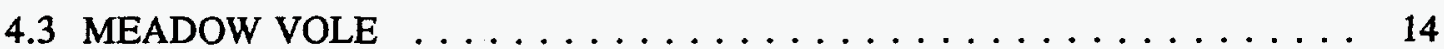

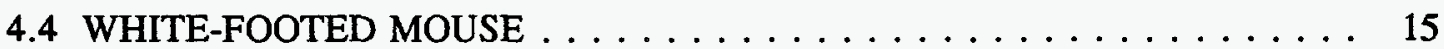

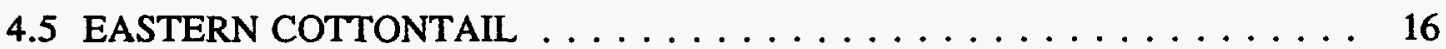

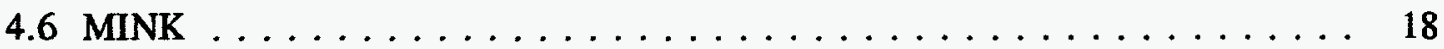

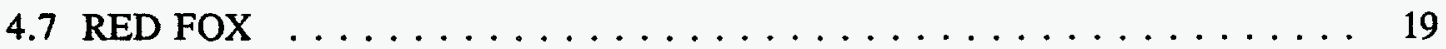

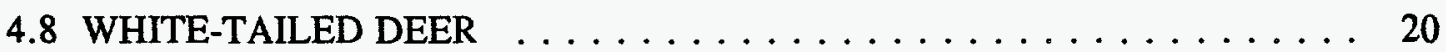

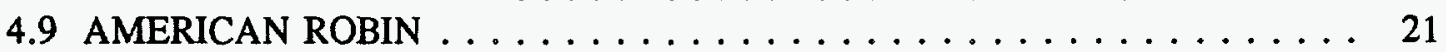

4.10 AMERICAN WOODCOCK $\ldots \ldots \ldots \ldots \ldots \ldots \ldots \ldots \ldots \ldots \ldots$

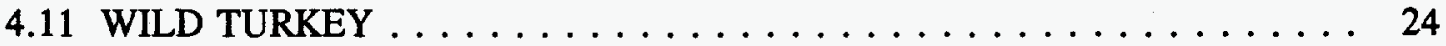

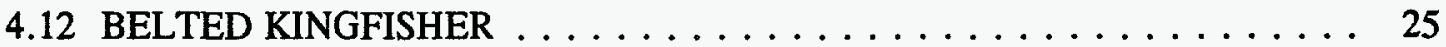

4.13 GREAT BLUE HERON $\ldots \ldots \ldots \ldots \ldots \ldots \ldots \ldots \ldots \ldots \ldots$

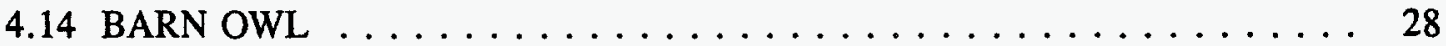

4.15 RED-TAILED HAWK . . . . . . . . . . . . . . . . . . . . . 29

5. APPLICATION OF THE MODELS $\ldots \ldots \ldots \ldots \ldots \ldots \ldots \ldots \ldots \ldots \ldots$

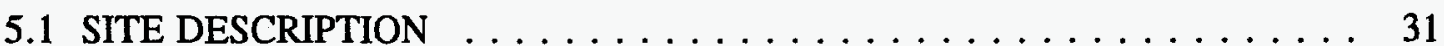

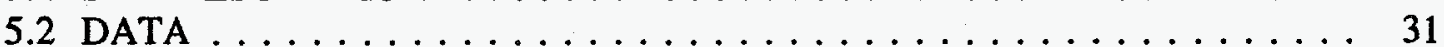

5.3 EXPOSURE ESTIMATION AND EVALUATION $\ldots \ldots \ldots \ldots \ldots \ldots \ldots$

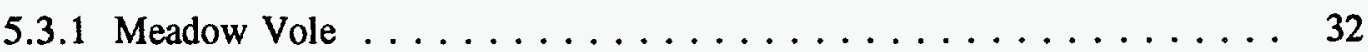

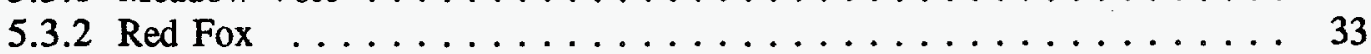

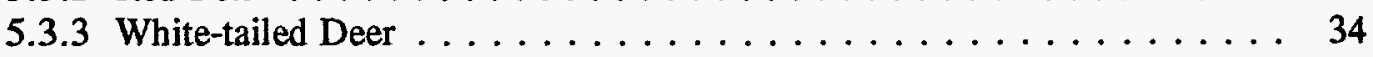

5.3.4 Red-tailed Hawk $\ldots \ldots \ldots \ldots \ldots \ldots \ldots \ldots \ldots$

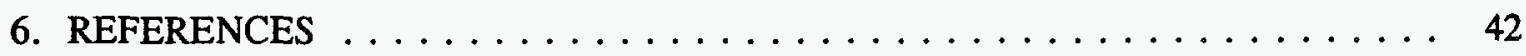




\section{TABLES}

Table 1. Percent water content of wildlife foods $\ldots \ldots \ldots \ldots \ldots \ldots$

Table 2. Estimation of contaminant exposure for meadow voles using the FCAP . . . . 36

Table 3. Estimation of contaminant exposure for red fox using the FCAP . . . . . 37

Table 4. Percent of total exposure attributed to medium for red fox using the FCAP . . . 38

Table 5. Estimation of contaminant exposure for white-tailed deer using the FCAP . . . 39

Table 6. Estimation of Contaminant Exposure for white-tailed deer consuming ash from the

FCAP to meet sodium needs $\ldots \ldots \ldots \ldots \ldots \ldots \ldots \ldots \ldots \ldots \ldots$ 


\section{ACRONYMS and ABBREVIATIONS}

$\begin{array}{ll}\text { DOE } & \text { United States Department of Energy } \\ \text { EPA } & \text { United States Environmental Protection Agency } \\ \text { FCAP } & \text { Filled Coal Ash Pond } \\ \text { GIS } & \text { Geographic Information System } \\ \text { NOAEL } & \text { No Observed Adverse Effects Level } \\ \text { ORR } & \text { Oak Ridge Reservation } \\ \text { UCL } & \text { Upper Confidence Limit } \\ \text { VOC } & \text { Volatile Organic Compound }\end{array}$




\section{ACKNOWLEDGMENTS}

This manuscript benefitted from the review comments of Tom Ashwood and Christopher Welsh. We are also grateful for the assistance of Kit Lash. 


\section{EXECUTIVE SUMMARY}

This report describes generalized models for the estimation of contaminant exposure experienced by wildlife on the Oak Ridge Reservation. The primary exposure pathway considered is oral ingestion, e.g. the consumption of contaminated food, water, or soil. Exposure through dermal absorption and inhalation are special cases and are not considered hereIN.

Because wildlife are mobile and generally consume diverse diets and because environmental contamination is not spatial homogeneous, factors to account for variation in diet, movement, and contaminant distribution have been incorporated into the models. To facilitate the use and application of the models, life history parameters necessary to estimate exposure are summarized for 15 common wildlife species. Finally, to display the application of the models, exposure estimates were calculated for four species using data from a source operable unit on the Oak Ridge Reservation. 


\section{INTRODUCTION}

This report presents a general model for exposure of terrestrial wildlife to contaminants (Sect. 2), methods for estimating parameters of the model (Sect. 3), species specific parameters for endpoint species on the Oak Ridge Reservation (ORR) (Sect. 4), and a sample application (Sect. 5). Exposure can be defined as the coincidence in both space and time of a receptor and a stressor, such that the receptor and stressor come into contact and interact (Risk Assessment Forum 1992). In the context of ecological risk assessment, receptors include all endpoint species or communities identified for a site [see Suter (1989) and Suter et al. (1994) for discussions of ecological endpoints for waste sites]. In the context of waste site assessments, stressors are chemical contaminations, and the contact and interaction are uptake of the contaminant by the receptor. Without sufficient exposure of the receptor to the contaminants, there is no ecological risk.

Unlike some other endpoint assemblages, terrestrial wildlife are significantly exposed to contaminants in multiple media. They may drink or swim in contaminated water, ingest contaminated food and soil, and breath contaminated air. In addition, because most wildlife are mobile, moving among and within habitats, exposure is not restricted to a single location. They may integrate contamination from several spatially discrete sources. Therefore, exposure models for terrestrial wildlife must include multiple media.

This document provides models and parameters for estimating exposure of birds and mammals. Reptiles and amphibians are not considered because few data exist with which to assess exposure to these organisms. In addition, because toxicological data are scarce for both classes, evaluation of the significance of exposure estimates is problematic. However, the general exposure estimation procedure developed herein for birds and mammals is applicable to reptiles and amphibians.

Exposure models must be appropriate to the assessment endpoints. The models presented herein are models of the exposure of individual organisms, but except for threatened and endangered species, all the wildlife endpoints for the ORR are for populations (Suter et al. 1994). The use of organism exposures is appropriate because of the need to integrate exposure estimates with exposure-response information which is expressed as organism-level responses. The conversion of individual exposure to population effects occurs in the risk characterization.

Conceptually, the conversion of organism-level exposures to the population level can be made in two ways. First, it may be assumed that there is a distinct population on the site so that the exposure of the population is the exposure of all the individuals. This assumption is appropriate for small organisms on large sites, particularly if the site constitutes a distinct habitat that is surrounded by inappropriate habitat. For example, a grassy site surrounded by forest or industrial development might support a distinct population of voles. The risks to that population can be estimated directly from the exposures of the individual organisms. Second, it may be assumed that a certain number of individuals are exposed to contaminants out of a larger population. For example, a certain proportion of a deer herd may forage on a site or a pair of hawks may hunt on a site. The estimated exposure of these individuals will result in estimation of certain effects on those individuals, and the resulting population risks will need to be characterized. In either case, the organism level exposure models are appropriate. 


\section{GENERALIZED EXPOSURE MODEL}

As terrestrial wildlife move through the environment, they may be exposed to contamination via three pathways: oral, dermal, or inhalation. Oral exposure occurs through the consumption of contaminated food, water, or soil. Dermal exposure occurs when contaminants are absorbed directly through the skin. Inhalation exposure occurs when volatile compounds or fine particulates are respired into the lungs. The total exposure experienced by an individual is the sum exposure from all three pathways or:

$$
E_{\text {total }}=E_{\text {oral }}+E_{\text {dermal }}+E_{\text {inhal }}
$$

where:

$$
\begin{aligned}
& E_{\text {ooal }}=\text { exposure from all pathways } \\
& E_{\text {oral }}=\text { oral exposure } \\
& E_{\text {dermal }}=\text { dermal exposure } \\
& E_{\text {inhal }}=\text { exposure through inhalation }
\end{aligned}
$$

Dermal exposure is assumed to be negligible for birds and mammals on most United States Department of Energy (DOE) waste sites. While methods are available to assess dermal exposure to humans (EPA 1992), data necessary to estimate dermal exposure are generally not available for wildlife (EPA 1993a). Additionally, many contaminants (e.g., metals and radionuclides) found on the ORR and other DOE facilities are unlikely to be absorbed through skin (Camner et al. 1979, Watters et al. 1980). Feathers and fur of birds and mammals further reduce the likelihood of significant dermal exposure by limiting the contact of skin with contaminated media. Therefore, dermal exposure is expected to be negligible relative to other routes in most cases and is not considered in the models presented in this report. If contaminants that have a high affinity for dermal uptake (e.g., organic solvents and pesticides) are present and an exposure scenario for an endpoint species is likely to result in significant dermal exposure (e.g., burrowing or swimming amphibians), then a research effort to quantify dermal exposure for those contaminants and species may be justified.

Inhalation of contaminants is also assumed to be negligible at the ORR and other DOE facilities for two reasons. (Therefore, the inhalation pathway is not considered in the models presented in this report.) First, because most contaminated sites are either capped or vegetated, exposure of contaminated surface soils to winds and resulting aerial suspension of contaminated dust particulates is minimized. Second, most volatile organic compounds (VOCs), the contaminants most likely to present a risk through inhalation exposure, rapidly volatilize from soil and surface water to air, where they are rapidly diluted and dispersed. Because of the age of the waste sites where VOCs were disposed of on the ORR and the short residence time of VOCs, significant exposure to volatile organic compounds through inhalation is unlikely. In situations where inhalation exposure of endpoint species is believed to be occurring or is expected to occur, methods to estimate the exposure and resulting risk should be developed. 
Because contaminant exposure experienced by wildlife through both the dermal and inhalation pathways is negligible, the majority of exposure must be attributed to the oral exposure pathway. Equation 1 can therefore be simplified to:

$$
\mathrm{E}_{\text {total }} \approx \mathrm{E}_{\text {oral }} \text {. }
$$

\subsection{ORAL EXPOSURE MODEL}

Oral exposure experienced by wildlife may come from multiple sources. They may consume contaminated food (either plant or animal), drink contaminated water, or ingest soil. Soil ingestion may be incidental while foraging or grooming or purposeful to meet nutrient needs. The total oral exposure experienced by an individual is the sum of the exposures attributable to each source and may be described as:

where:

$$
E_{\text {total }} \approx E_{\text {oral }}+E_{\text {food }}+E_{\text {water }}+E_{\text {soil }}
$$

$$
\begin{aligned}
& \mathrm{E}_{\text {roal }}=\text { total exposure from all pathways } \\
& \mathrm{E}_{\text {oral }}=\text { total exposure from oral ingestion } \\
& \mathrm{E}_{\text {food }}=\text { exposure from food consumption } \\
& \mathrm{E}_{\text {water }}=\text { exposure from water consumption } \\
& \mathrm{E}_{\text {soil }}=\text { exposure from soil consumption }
\end{aligned}
$$

For exposure estimates to be useful in the assessment of risk to wildlife, they must be expressed in terms of a body weight-normalized daily dose or $\mathrm{mg}$ contaminant per $\mathrm{kg}$ body weight per day $(\mathrm{mg} / \mathrm{kg} / \mathrm{d})$. Exposure estimates expressed in this manner may then be compared to toxicological benchmarks for wildlife, such as those derived by Opresko et al. (1994), or to doses reported in the toxicological literature. Estimation of the daily contaminant dose an individual may receive from a particular medium for a particular contaminant may be calculated using the following equation:

$$
E_{j}=\sum_{i=1}^{m}\left(\frac{I R_{i} \times C_{i j}}{B W}\right)
$$

where:

$\mathrm{E}_{\mathrm{j}} \quad=$ total exposure to contaminant $(\mathrm{j})(\mathrm{mg} / \mathrm{kg} / \mathrm{d})$

$\mathrm{m}=$ total number of ingested media (e.g., food, water, or soil)

$\mathrm{IR}_{\mathrm{i}} \quad=$ consumption rate for medium (i) $(\mathrm{kg} / \mathrm{d}$ or $\mathrm{L} / \mathrm{d})$

$\mathrm{C}_{\mathrm{ij}}=$ concentration contaminant $(\mathrm{j})$ in medium (i) $(\mathrm{mg} / \mathrm{kg}$ or $\mathrm{mg} / \mathrm{L})$

$\mathrm{BW}=$ body weight of endpoint species $(\mathrm{kg})$

Note: Soil ingestion rates and soil contaminant concentrations are in kg dry weight. All other weights (body weights, food, etc.) are in $\mathrm{kg}$ fresh weight. 


\subsection{DIET COMPOSITION}

Few wildlife species consume diets that consist exclusively of one food type. To meet nutrient needs for growth, maintenance, and reproduction, most wildlife consume varying amounts of multiple food types. Because it is unlikely that all food types consumed will contain the same contaminant concentrations, dietary diversity is of one of the most important exposure modifying factors.

To account for varying contaminant concentrations in different food types, exposure estimates should be weighted by the relative proportion of daily food consumption attributable to each food type and the contaminant concentration in each food type. This may be done by modifying Equation 4 as follows:

$$
F E_{j}=\sum_{i=1}^{m}\left(\frac{F I R_{i} \times C_{i j}}{B W}\right)
$$

where:

$\mathrm{C}_{\mathrm{ij}}=$ concentration of contaminant (j) in food type (i) (mg/kg, fresh weight)

$\mathrm{FE}_{\mathrm{j}}=$ exposure to contaminant (j) attributed to food $(\mathrm{mg} / \mathrm{kg} / \mathrm{d})$

$\mathrm{FIR}_{\mathrm{i}}=$ ingestion rate ( $\mathrm{kg} /$ individual/day) for the $\mathrm{i}^{\text {th }}$ food type

The ingestion rate for each food type, $\mathrm{FIR}_{\mathrm{i}}$, may be estimated as follows:

$$
F I R_{i}=P_{i} x F I R
$$

where:

$P_{i} \quad=$ proportion of the $i^{\text {th }}$ food type in the diet

FIR = total food ingestion rate $(\mathrm{kg} /$ individual/day)

\subsection{HOME RANGE/HABITAT REQUIREMENTS}

If the site is spatially heterogeneous with respect to either contamination or wildlife use, the model must be modified to include spatial factors. The most important spatial consideration is the movement of wildlife. Animals travel varying distances, on a daily to seasonal basis, to find food, water, and shelter. The area encompassed by these travels is defined as the home range. (The term is used herein to include territories. A territory is a subset of the home range that is actively defended to exclude other con-specific individuals.) If the site being assessed is larger than the home range of an endpoint species and provides the habitat needs of the species, then the previously listed models are adequate. However, endpoint species often have home ranges that are larger than contaminated sites, or the contaminated site may not supply all of a species habitat requirements. In those cases, the wildlife exposure model must be modified to account for the fraction of the home range potentially encompassed by suitable habitat at the site. 
If the contaminated site has similar habitat quality to the surrounding area but is smaller than the home range, use of the contaminated site is simply a function of its area. That is, one can assume that exposure is proportional to the ratio of the size of the contaminated site to home range size and modify Equation 4 as follows:

$$
E_{j}=\left(\frac{A}{H R}\left[\sum_{i=1}^{m}\left(\frac{I R_{i} \times C_{i j}}{B W}\right)\right]\right)
$$

where:

$\mathrm{A}=$ area (ha) contaminated

$\mathrm{HR}=$ home range size (ha) of endpoint species

Note that $A$ is the area contaminated, not the entire area that has been designated a waste site (e.g., an operable unit). Because boundaries are often drawn conservatively, they may contain a considerable uncontaminated area. However, because more detailed characterization of contaminant distribution is generally not available, assuming that the entire area is contaminated may be appropriate. To prevent grossly inflated estimates, in cases where the size of the contaminated area is greater than the organism's home range, A/HR should be set to 1 .

The previous equation (7) implies that all the habitat within a contaminated area is suitable, and use of all portions of the contaminated area is equally likely. Because many waste sites are industrial or highly modified in nature, it is unlikely that all areas within their bounds will provide habitat suitable for endpoint species. If it is assumed that use of a waste site will be proportional to the amount of suitable habitat available on the site, Equation 7 may be modified to read:

$$
E_{j}=P_{h}\left(\frac{A}{H R}\left[\sum_{i=1}^{m}\left(\frac{I R_{i} \times C_{i j}}{B W}\right)\right]\right)
$$

where:

$\mathrm{P}_{\mathrm{h}} \quad=$ proportion of suitable habitat in the contaminated area.

One complication is the spatial heterogeneity of contaminants on waste sites. These models (Equations 4-8) are based on the assumption that either contaminants are evenly distributed on the site or that wildlife forage randomly with respect to contamination on the portion of the site which constitutes habitat so wildlife are exposed to mean concentrations. However, if contaminant levels are related to habitat quality, that assumption would not hold. For example, contaminant concentrations might be greatest near the center of a site, but the habitat quality might be highest near the edges. In such cases, it might be necessary to model the proportional contribution of each area with a distinct combination of contaminant level and habitat quality. 


$$
E_{j}=\sum_{k=1}^{n}\left(\frac{A_{k}}{H R}\left[\sum_{i=1}^{m}\left(\frac{I R_{i} x C_{i j k}}{B W}\right)\right]\right)
$$

where:

$\mathrm{n}=$ number of distinct contaminated habitat areas

$\mathrm{A}_{\mathrm{k}} \quad=$ area (ha) of a distinct contaminated habitat area

$\mathrm{C}_{\mathrm{ijk}}=$ concentration of contaminant $(\mathrm{j})$ in the $\mathrm{i}^{\text {th }}$ medium type in the $\mathrm{k}^{\text {th }}$ area $(\mathrm{mg} / \mathrm{kg}$ or $\mathrm{mg} / \mathrm{L}$ )

To model exposures of wide-ranging wildlife to multiple waste sites, as is planned for the ORR (Suter et al. 1994), Equation 9 should be modified to include multiple waste sites as follows:

$$
E_{j}=\sum_{l=1}^{o} \sum_{k=1}^{n}\left(\frac{A_{k l}}{H R}\left[\sum_{i=1}^{m}\left(\frac{I R_{i} \times C_{i j k l}}{B W}\right)\right]\right)
$$

where:

$0=$ number of waste sites

$\mathrm{A}_{\mathrm{kd}} \quad=$ area (ha) of a distinct contaminated habitat area within each waste site

$\mathrm{C}_{\mathrm{ijd}}=$ concentration of contaminant $(\mathrm{j})$ in the $\mathrm{i}^{\text {th }}$ medium type in the $\mathrm{k}^{\text {th }}$ habitat area of the $\mathrm{l}^{\text {th }}$ waste site $(\mathrm{mg} / \mathrm{kg}$ or $\mathrm{mg} / \mathrm{L})$

If the distribution of contamination and habitat quality is complex, this approach becomes ungainly. In such cases, it is advisable to implement the exposure in a Geographic Information System (GIS). Using a GIS, maps displaying the spatial distribution of various habitat types may be overlaid with maps of contaminant distribution to accurately determine the degree to which habitat is contaminated. Furthermore, if information on the distribution or movements of wildlife (generated by radiotelemetry or censuses) are available, these data may be combined with the habitat and contamination data to provide a more accurate visualization of exposure.

\subsection{OTHER FACTORS}

Factors other than those described in these models modify contaminant exposure experienced by wildlife endpoint species. These factors include age, sex, season, and behavior patterns.

These models imply that the endpoint species have uniform body size, metabolism, diet, home ranges, and habitat requirements. However, these properties may differ between juveniles and adults, and males and females. For example, because they are actively growing, metabolism (and therefore the rate of food consumption) is generally greater for juveniles for most endpoint species. Similarly, the food requirements of females during reproduction are greater than that for males for many endpoint species. These factors may serve to make certain age classes or a particular sex experience greater contaminant exposure than other segments of the population. Because of their greater exposure, contamination may present a greater risk to these segments of 
the population. If it is known that a particular lifestage or sex is sensitive to contamination, that lifestage should be emphasized in the exposure assessment.

Behavior may modify exposure by increasing or decreasing the likelihood of contact with contaminated media. Wildlife behaviors are frequently seasonal in nature. Some foods may be available and consumed only at certain times of the year. Similarly, some habitats and certain parts of the home range may be used only in certain seasons. In addition, many species hibernate or migrate; by leaving the area or restricting their activity to certain times of year, their potential exposure may be dramatically reduced. All of these factors should be considered when evaluating contaminant exposure experienced by wildlife, and exposure models should be adjusted accordingly.

\section{PARAMETERS FOR ESTIMATION OF EXPOSURE}

Implementation of the exposure model presented in Equation 4 requires the specification of certain parameters. Some parameters, such as home range size, body weights (Sect. 3.1), and soil consumption rates (Sect. 3.3), must be obtained from the literature for the endpoint species, while others, such as area contaminated, are site-specific. General methods are available for estimating food and water consumption rates (Sects. 3.2 to 3.4 ).

\subsection{BODY WEIGHT}

Body weight is an important parameter in the estimation of exposure. Not only is it a factor in determining the exposure rate (see Equation 4), but because metabolism and body weight are related, body weights may be used to predict food and water consumption rates. On an individual basis, larger animals consume more food or water than do smaller animals. However, larger animals have lower metabolic rates than smaller animals because smaller bodies have higher food and water consumption rates per unit body weight. Therefore, smaller animals will experience greater oral exposure per unit body weight than will larger animals.

Body weights for selected terrestrial wildlife are reported by the United States Environmental Protection Agency (EPA) (1993a). Additional sources include Dunning (1984), Burt and Grossenheider (1976), and the Mammalian Species series, published by the American Society of Mammalogists.

\subsection{FOOD AND WATER CONSUMPTION}

Field observations of food, water, or soil consumption rates are the best data to use to estimate exposure. With few exceptions, these data are unavailable for wildlife species. The second best data to use to estimate exposure are media consumption rates for wildlife species derived from laboratory studies. These data are limited because the influence of ambient conditions, such as activity regimes or environmental variables (temperature, humidity, etc.), on metabolism (and therefore consumption rates) are difficult to approximate in a laboratory setting. 
In the absence of experimental data, food consumption values can be estimated from allometric regression models based on metabolic rate. Nagy (1987) derived equations to estimate food consumption (in kg dry weight) for various groups of birds and mammals:

$$
\begin{aligned}
& F I R=0.0687(B W)^{0.822} \quad \text { (placental mammals) } \\
& F I R=0.0306(B W)^{0.564} \quad \text { (rodents) } \\
& F I R=0.0875(B W)^{0.727} \quad \text { (herbivores) } \\
& F I R=0.0514(B W)^{0.673} \quad \text { (marsupials) } \\
& F I R=0.0582(B W)^{0.651} \quad \text { (all birds) } \\
& F I R=0.141(B W)^{0.850} \quad \text { (passerine birds) }
\end{aligned}
$$

Note: $\mathrm{BW}$ is in $\mathrm{kg}$ fresh or live weight.

Food ingestion rates estimated using these allometric equations are expressed as kg dry weight. Because wildlife do not generally consume dry food (unless being maintained in the laboratory), food consumption must be converted to $\mathrm{kg}$ fresh weight by adding the water content of the food. Percent water content of wildlife foods are listed in Table 1. Calculation of food consumption in $\mathrm{kg}$ fresh weight is performed as follows:

$$
F I R_{\text {fresh }}=\sum_{i=1}^{f}\left(P_{i} \times \frac{F I R_{d r y}}{1-W C_{i}}\right)
$$

where:

$$
\begin{array}{ll}
\text { FIR }_{\text {fresh }} & =\text { total food ingestion rate (kg food [fresh weight]/individual/day) } \\
\mathbf{f} & =\text { total number of food types in the diet } \\
\mathbf{P}_{\mathrm{i}} & =\text { proportion of the } \mathrm{i}^{\text {th }} \text { food type in the diet } \\
\mathrm{WC}_{\mathrm{i}} & =\text { proportional water content (by weight) of the } \mathrm{i}^{\text {th }} \text { food type }
\end{array}
$$


9

Table 1. Percent water content of wildlife foods

\begin{tabular}{|c|c|c|c|}
\hline \multirow{2}{*}{ Food Type } & \multicolumn{3}{|c|}{ Percent Water Content } \\
\hline & Mean & $\begin{array}{l}\text { Standard } \\
\text { Deviation }\end{array}$ & Range $^{b}$ \\
\hline \multicolumn{4}{|c|}{ Aquatic Invertebrates } \\
\hline bivalves (w/o shell) & 82 & 4.5 & \\
\hline crabs (w/shell) & 74 & 6.1 & \\
\hline shrimp & 78 & 3.3 & \\
\hline isopods, amphipods & & & $71-80$ \\
\hline cladocerans & & & $79-87$ \\
\hline \multicolumn{4}{|c|}{ Aquatic Vertebrates } \\
\hline bony fishes & 75 & 5.1 & \\
\hline Pacific herring & 68 & 3.9 & \\
\hline \multicolumn{4}{|c|}{ Aquatic Plants } \\
\hline algae & 84 & 4.7 & \\
\hline aquatic macrophytes & 87 & 3.1 & \\
\hline emergent vegetation & & & $45-80$ \\
\hline \multicolumn{4}{|c|}{ Terrestrial Invertebrates } \\
\hline earthworms (depurated) & 84 & 1.7 & \\
\hline grasshoppers, crickets & 69 & 5.6 & \\
\hline beetles (adult) & 61 & 9.8 & \\
\hline \multicolumn{4}{|c|}{ Mammals } \\
\hline mice, voles, rabbits & 68 & 1.6 & \\
\hline \multicolumn{4}{|c|}{ Birds } \\
\hline passerines (w/typical fat reserves) & & & 68 \\
\hline mallard duck (flesh only) & & & 67 \\
\hline \multicolumn{4}{|c|}{ Reptiles and Amphibians } \\
\hline snakes, lizards & & & 66 \\
\hline frogs,toads & 85 & 4.7 & \\
\hline \multicolumn{4}{|c|}{ Terrestrial Plants } \\
\hline monocots: young grass & & & $70-88$ \\
\hline
\end{tabular}


10

Table 1. (continued)

\begin{tabular}{lccc} 
& Food Type & \multicolumn{3}{c}{ Percent Water Content } \\
\cline { 2 - 4 } & Mean & $\begin{array}{c}\text { Standard } \\
\text { Deviation }\end{array}$ & Range $^{\mathrm{b}}$ \\
\hline monocots: mature dry grass & & & $7-10$ \\
dicots: leaves & 85 & 3.5 & \\
dicots: seeds & 9.3 & 3.1 & \\
fruit: pulp, skin & 77 & 3.6 & \\
\hline
\end{tabular}

Derived from EPA 1993a

b Single values indicate only one value available.

Water consumption rates can also be estimated for mammals and birds from allometric regression models based on body weight (Calder and Braun 1983):

$$
W I R=0.099(B W)^{0.90} \text { mammals }
$$

$$
W I R=0.059(B W)^{0.67} \text { birds }
$$

where:

WIR = water ingestion rate ( $\mathrm{L}$ water/individual/day).

Note: $\mathrm{BW}$ is in $\mathrm{kg}$ fresh or live weight.

\subsection{SOIL CONSUMPTION}

In addition to consuming food and water, many wildlife consume soil. Soil consumption may occur inadvertently while foraging (i.e., predators of soil invertebrates ingesting soil adhering to worms, grazing herbivores consuming soil deposited on foliage or adhering to roots, etc.) or grooming or purposefully to meet nutrient requirements because diets of many herbivores are deficient in sodium and other trace nutrients (Robbins 1993). For example, ungulates, such as white-tailed deer (Odocoileus virginianus), have been observed to consume soils with elevated sodium levels, presumably to meet sodium needs (Weeks 1978). Because soils at waste sites may contain high contaminant concentrations, direct ingestion of soil is potentially a significant exposure source. In contrast to food and water consumption, generalized models do not exist with which to estimate soil ingestion by wildlife. Beyer et al. (1994) reports soil consumption estimates for 28 wildlife species. Additional data concerning soil consumption are reported in Arthur and Alldredge (1979), Garten (1980), Thornton and Abrahams (1983), and Arthur and Gates (1988), 


\section{LIFE HISTORY PARAMETERS FOR SELECTED ENDPOINT SPECIES}

To estimate contaminant exposure to terrestrial wildlife using the models described previously, species-specific values for the parameters are needed. Due to the large within-species variation in values for life-history parameters, data specific to the site in question would provide the most accurate exposure estimates. However, these data are virtually never available. Published values from other areas within an endpoint species range must therefore be used to estimate life-history parameters. Life-history parameters used in determining contaminant exposure are listed in the following tables for eight mammals and seven birds likely to be present on the ORR. These values were selected to be representative of each endpoint species, but it is recognized that some species display a high degree of variation for some parameters. Additional, more detailed data may be found in EPA (1993a) or obtained by searching the open literature if necessary.

\subsection{SHORT-TAILED SHREW (Blarina brevicauda)}

\begin{tabular}{|c|c|c|c|}
\hline Parameter & Value $^{2}$ & Comments & Reference \\
\hline Body Weight & $0.015 \pm 0.00078 \mathrm{~kg}$ & New Hampshire (field) & $\begin{array}{l}\text { Schlessinger and Potter } \\
1974\end{array}$ \\
\hline Food Consumption Rate & $\begin{array}{l}0.01 \mathrm{~kg} / \mathrm{d} \\
0.00795 \pm 0.00017 \mathrm{~kg} / \mathrm{d} \\
\text { mean }=0.009 \mathrm{~kg} / \mathrm{d}\end{array}$ & $\begin{array}{l}\text { larch sawfly diet (lab) } \\
\text { mealworm diet (lab) }\end{array}$ & $\begin{array}{l}\text { Buckner } 1964 \\
\text { Barrett and Stueck } 1976\end{array}$ \\
\hline Water Consumption Rate & $\begin{array}{l}0.223 \mathrm{ml} / \mathrm{g} \mathrm{bw} / \mathrm{d} \\
0.0033 \mathrm{~L} / \mathrm{d}\end{array}$ & assuming a $0.015 \mathrm{~kg} \mathrm{bw}$ & Chew 1951 \\
\hline Soil Consumption Rate ${ }^{\mathfrak{c}}$ & $\begin{array}{l}13 \% \text { of diet } \\
0.00117 \mathrm{~kg} / \mathrm{d}\end{array}$ & $\begin{array}{l}\text { assuming diet of } 0.009 \\
\mathrm{~kg} / \mathrm{d}\end{array}$ & $\begin{array}{l}\text { Talmage and Walton } \\
1993\end{array}$ \\
\hline Diet Composition & $\begin{array}{l}\text { earthworms } 31.4 \% \\
\text { slugs/snails } 27.1 \% \\
\text { soil/litter invert } 13.2 \% \\
\text { fungi } 8.4 \% \\
\text { misc. animals } 8.1 \% \\
\text { coleoptera } 5.9 \% \\
\text { vegetation } 5.4 \%\end{array}$ & $\begin{array}{l}\text { percent volume in diet in } \\
\text { summer in New York }\end{array}$ & $\begin{array}{l}\text { Whitaker and Ferraro } \\
1963\end{array}$ \\
\hline Home Range & $0.39 \pm 0.036 \mathrm{ha}$ & Manitoba bog & Buckner 1966 \\
\hline
\end{tabular}


Short-tailed Shrew (continued)

\begin{tabular}{|c|c|c|c|}
\hline Parameter & Value $^{2}$ & Comments & Reference \\
\hline Habitat Requirements & $\begin{array}{l}\text { broad and variable but } \\
\text { requires }>50 \% \\
\text { herbaceous cover } \\
\text { forest, wetlands, and } \\
\text { grasslands. Most } \\
\text { abundant in hardwood } \\
\text { forests with deep litter } \\
\text { and humus. }\end{array}$ & & $\begin{array}{l}\text { Miller and Getz } 1977 \\
\text { van Zyll de Jong } 1983\end{array}$ \\
\hline Population Density & $\begin{array}{l}2.3 \text { ha - winter } \\
5.2 \text { ha - spring } \\
9.3 \text { ha -summer } \\
8.1 \text { ha - fall }\end{array}$ & $\begin{array}{l}\text { Illinois - alfalfa, } \\
\text { tallgrass, and bluegrass; } \\
\text { means derived from } \\
\text { graph. }\end{array}$ & Getz 1989 \\
\hline \multirow[t]{2}{*}{ Behavior } & $\begin{array}{l}\text { nocturnal, semifossorial, } \\
\text { spends little time above } \\
\text { surface }\end{array}$ & & George et al. 1986 \\
\hline & $\begin{array}{l}\text { active year-round - does } \\
\text { not hibernate }\end{array}$ & & EPA 1993a \\
\hline Other & $\begin{array}{l}\text { appear to be unpalatable } \\
\text { to most predators due to } \\
\text { lateral gland }\end{array}$ & & van Zyll de Jong 1983 \\
\hline
\end{tabular}

'Suggested values for use in exposure assessment are in bold.

bg/d wet weight

c $\mathrm{kg} / \mathrm{d}$ dry weight

\subsection{LITTLE BROWN BAT (Myotis lucifugus)}

\begin{tabular}{|c|c|c|c|}
\hline Parameter & Value $^{2}$ & Comments & Reference \\
\hline Body Weight & $0.0075 \mathrm{~kg}$ & & Gould 1955 \\
\hline \multirow[t]{3}{*}{ Food Consumption Rate } & $0.0025 \mathrm{~kg} / \mathrm{d}$ & pregnant $q$ (field) & Anthony and Kunz 1977 \\
\hline & $0.0037 \mathrm{~kg} / \mathrm{d}$ & lactating $ㅇ$ (field) & \\
\hline & $0.0018 \mathrm{~kg} / \mathrm{d}$ & juvenile (field) & \\
\hline Water Consumption Rate & $0.0012 \mathrm{~L} / \mathrm{d}$ & $\begin{array}{l}\text { estimated using } \\
\text { Equation } 18 ; \\
\text { assuming } 0.0075 \mathrm{~kg} \mathrm{bw}\end{array}$ & \\
\hline
\end{tabular}

Soil Consumption Rate ${ }^{c}$ as an aerial insectivore, assumed to be negligible 
Little Brown Bat (continued)

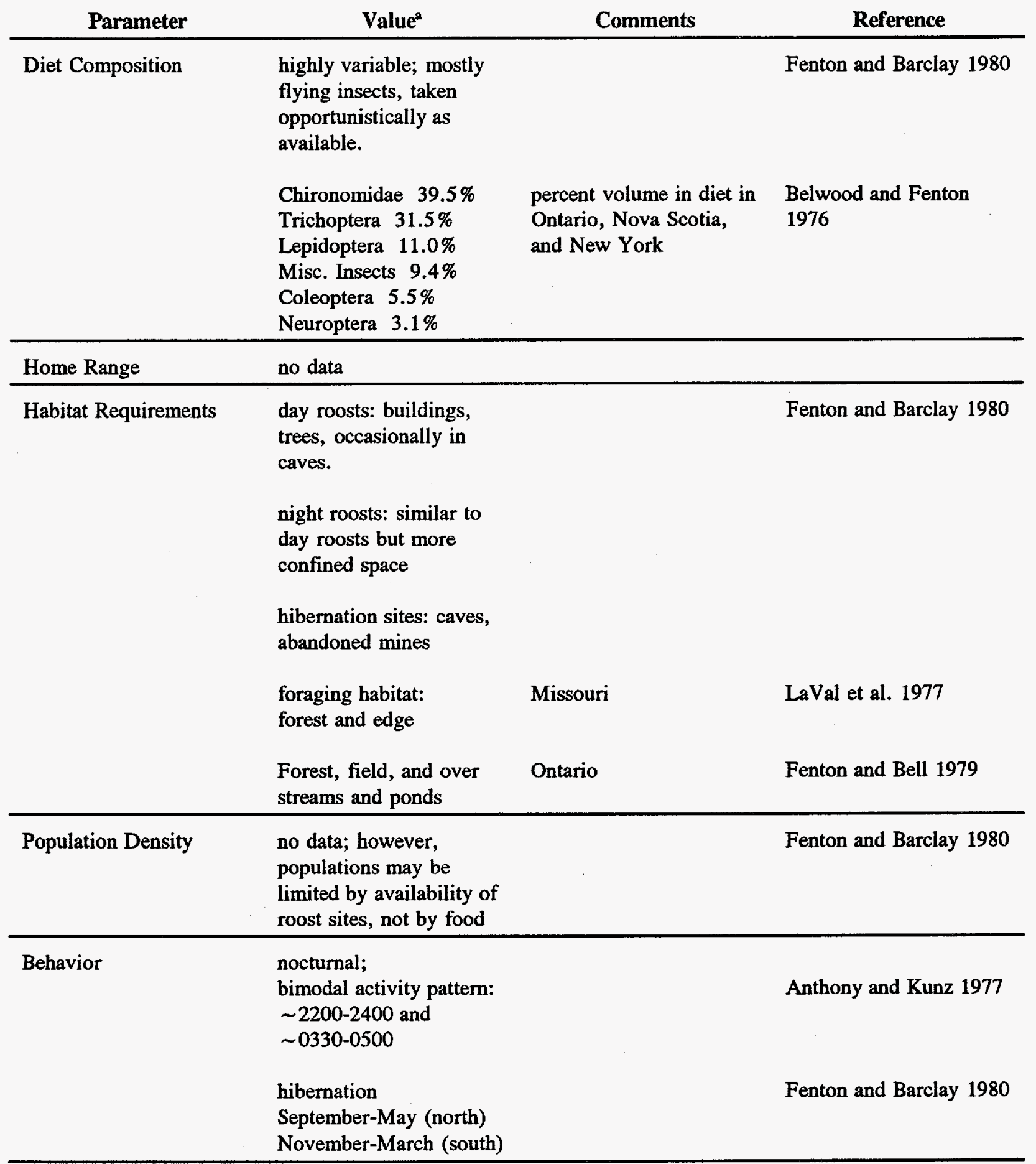


Little Brown Bat (continued)

\begin{tabular}{|c|c|c|c|}
\hline Parameter & Value $^{a}$ & Comments & Reference \\
\hline Other & $\begin{array}{l}\text { long-lived; may live up } \\
\text { to } 30 \mathrm{yrs} \text {. } \\
\text { mean life expectancy: } \\
\delta=4.9 \mathrm{yrs} \\
\$=2.9 \mathrm{yrs}\end{array}$ & & $\begin{array}{l}\text { Keen and Hitchcock, } \\
1980\end{array}$ \\
\hline
\end{tabular}

Suggested values for use in exposure assessment are in bold.

${ }^{b} \mathrm{~kg} / \mathrm{d}$ wet weight

${ }^{c} \mathrm{~kg} / \mathrm{d}$ dry weight

\subsection{MEADOW VOLE (Microtus pennsylvanicus)}

\begin{tabular}{|c|c|c|c|}
\hline Parameter & Value $^{\mathrm{a}}$ & Comments & Reference \\
\hline Body Weight & $0.044 \mathrm{~kg}$ & & Reich 1981 \\
\hline Food Consumption Rate ${ }^{b}$ & $0.005 \mathrm{~kg} / \mathrm{d}$ & $\begin{array}{l}\text { estimated from figure } \\
\text { (lab) }\end{array}$ & Dark et al. 1983 \\
\hline Water Consumption Rate & $0.006 \mathrm{~L} / \mathrm{d}$ & $\begin{array}{l}\text { estimated using } \\
\text { Equation } 18 ; \\
\text { assuming } 0.044 \mathrm{~kg} \mathrm{bw}\end{array}$ & \\
\hline Soil Consumption Rate & $\begin{array}{l}2.4 \% \text { of diet } \\
0.00012 \mathrm{~kg} / \mathrm{d}\end{array}$ & $\begin{array}{l}\text { assuming diet of } 0.005 \\
\mathrm{~kg} / \mathrm{d}\end{array}$ & Beyer et al. 1994 \\
\hline Diet Composition & $\begin{array}{l}\text { herbivorous } \\
\text { predominantly monocot } \\
\text { and dicot shoots; lesser } \\
\text { amounts of seeds and } \\
\text { roots (more in winter); } \\
\text { minimal fungi and } \\
\text { insects }\end{array}$ & $\begin{array}{l}\text { Illinois bluegrass and } \\
\text { prairie habitat }\end{array}$ & Lindroth and Batzli 1984 \\
\hline Home Range & $\begin{array}{l}0.083 \pm 0.037 \text { ha } \delta \\
0.037 \pm 0.020 \text { ha } 9\end{array}$ & $\begin{array}{l}\text { Massachusetts - grassy } \\
\text { meadow }\end{array}$ & Ostfeld et al. 1988 \\
\hline Habitat Requirements & $\begin{array}{l}\text { grassy fields, marshes, } \\
\text { and bogs }\end{array}$ & & Getz 1961 \\
\hline
\end{tabular}


Meadow Vole (continued)

\begin{tabular}{|c|c|c|c|}
\hline Parameter & Value $^{2}$ & Comments & Reference \\
\hline \multirow[t]{4}{*}{ Population Density } & $28-85 / \mathrm{ha}$ & $\begin{array}{l}\text { Massachusetts - grassy } \\
\text { meadow }\end{array}$ & Ostfeld et al. 1988 \\
\hline & $\sim 8-\sim 20 / \mathrm{ha}$ & Michigan - old field & Getz 1961 \\
\hline & $2-28 / \mathrm{ha}$ & Illinois bluegrass & Lindroth and Batzli 1984 \\
\hline & $26-128 / \mathrm{ha}$ & Illinois prairie & \\
\hline \multirow[t]{2}{*}{ Behavior } & $\begin{array}{l}\text { may be either diurnal or } \\
\text { nocturnal; activity } \\
\text { depends on amount of } \\
\text { vegetative cover }\end{array}$ & & Reich 1981 \\
\hline & $\begin{array}{l}\text { active year-round-does } \\
\text { not hibernate }\end{array}$ & & EPA 1993a \\
\hline Other & $\begin{array}{l}\text { vole population } \\
\text { decreases as short-tailed } \\
\text { shrew numbers increase }\end{array}$ & & Eadie 1952 \\
\hline \multicolumn{4}{|c|}{$\begin{array}{l}\text { Suggested values for use in exposure assessment are in bold. } \\
6 \mathrm{~kg} / \mathrm{d} \text { wet weight } \\
{ }^{\circ} \mathrm{kg} / \mathrm{d} \text { dry weight }\end{array}$} \\
\hline \multicolumn{4}{|c|}{ 4.4 WHITE-FOOTED MOUSE (Peromyscus leucopus) } \\
\hline Parameter & Value $^{\mathbf{2}}$ & Comments & Reference \\
\hline Body Weight & $0.022 \mathrm{~kg}$ & & Green and Millar 1987 \\
\hline Food Consumption Rate ${ }^{\mathrm{b}}$ & $0.0034 \mathrm{~kg} / \mathrm{d}$ & lab study & Green and Millar 1987 \\
\hline Water Consumption Rate & $0.0066 \mathrm{~L} / \mathrm{d}$ & 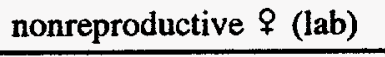 & Oswald et al. 1993 \\
\hline \multirow[t]{2}{*}{ Soil Consumption Rate } & $<2 \%$ & & Beyer et al. 1994 \\
\hline & $0.000068 \mathrm{~kg} / \mathrm{d}$ & $\begin{array}{l}\text { assuming diet of } 0.0034 \\
\mathrm{~kg} / \mathrm{d} \text { and a } 2 \% \text { soil } \\
\text { consumption rate }\end{array}$ & \\
\hline
\end{tabular}


White-footed Mouse (continued)

\begin{tabular}{|c|c|c|c|}
\hline Parameter & Value $^{2}$ & Comments & Reference \\
\hline \multirow[t]{4}{*}{ Diet Composition } & $\begin{array}{l}\text { omnivorous and } \\
\text { opportunistic }\end{array}$ & & \\
\hline & $\begin{array}{l}\text { arthropods- } 57 \% \\
\text { seeds, fruit, } \\
\text { vegetation-34\% }\end{array}$ & Virginia & Wolff et al. 1985 \\
\hline & $\begin{array}{l}\text { arthropods-30\% } \\
\text { seeds, fruit, } \\
\text { vegetation-67\% }\end{array}$ & Indiana & Whitaker 1966 \\
\hline & $\begin{array}{l}\text { arthropods- } 50 \% \\
\text { seeds, fruit, } \\
\text { vegetation- }-48 \%\end{array}$ & Illinois & Batzli 1977 \\
\hline Home Range & 0.059 ha & $\begin{array}{l}\text { mean: } \delta+q ; \text { Virginia, } \\
\text { mixed deciduous forest }\end{array}$ & Wolff 1985 \\
\hline Habitat Requirements & $\begin{array}{l}\text { wooded, brushy areas; } \\
\text { sometimes open areas }\end{array}$ & & $\begin{array}{l}\text { Burt and Grossenheider } \\
1976\end{array}$ \\
\hline Population Density & $6-57 / \mathrm{ha}$ & $\begin{array}{l}\text { Virginia, mixed } \\
\text { deciduous forest }\end{array}$ & Wolff 1985 \\
\hline \multirow[t]{3}{*}{ Behavior } & $\begin{array}{l}\text { while semi-arboreal, } \\
\text { spends most of time on } \\
\text { ground. }\end{array}$ & & Lackey et al. 1985 \\
\hline & primarily nocturnal & & \\
\hline & $\begin{array}{l}\text { enters torpor to reduce } \\
\text { metabolic demands in } \\
\text { winter and during food } \\
\text { stress }\end{array}$ & & EPA 1993a \\
\hline
\end{tabular}

2 Suggested values for use in exposure assessment are in bold.

b $\mathrm{kg} / \mathrm{d}$ wet weight

- $\mathrm{kg} / \mathrm{d}$ dry weight

\subsection{EASTERN COTTONTAIL (Sylvilagus floridanus)}

\begin{tabular}{|c|c|c|c|}
\hline Parameter & Value $^{2}$ & Comments & Reference \\
\hline Body Weight & $\begin{array}{l}1.134 \mathrm{~kg}\left({ }^{\circ}\right) \\
1.244 \mathrm{~kg}(q) \\
1.2 \mathrm{~kg}\left(\text { mean } \delta^{\circ}+\uparrow\right)\end{array}$ & & Chapman et al. 1980 \\
\hline Food Consumption Rate ${ }^{b}$ & $0.237 \mathrm{~kg} / \mathrm{d}$ & & Dalke and Sime 1941 \\
\hline
\end{tabular}


Eastern Cottontail (continued)

\begin{tabular}{|c|c|c|c|}
\hline Parameter & Value $^{2}$ & Comments & Reference \\
\hline Water Consumption Rate & $0.116 \mathrm{~L} / \mathrm{d}$ & $\begin{array}{l}\text { estimated using } \\
\text { Equation } 18 ; \\
\text { assuming } 1.2 \mathrm{~kg} \mathrm{bw}\end{array}$ & \\
\hline \multirow[t]{2}{*}{ Soil Consumption Rate } & & $\begin{array}{l}\text { assumed comparable to } \\
\text { that for black-tailed } \\
\text { jackrabbit ( } 6.3 \% \text { of diet) }\end{array}$ & Arthur and Gates 1988 \\
\hline & $0.015 \mathrm{~kg} / \mathrm{d}$ & $\begin{array}{l}\text { assuming diet of } 0.237 \\
\mathrm{~kg} / \mathrm{d} \text { and a } 6.3 \% \text { soil } \\
\text { consumption rate }\end{array}$ & \\
\hline
\end{tabular}

\begin{tabular}{|c|c|c|c|}
\hline Diet Composition & $\begin{array}{l}\text { exclusively herbivorous } \\
\text { bark, twigs, buds, dried } \\
\text { herbs (fall, winter) } \\
\text { herbaceous plants } \\
\text { (spring, summer) }\end{array}$ & & Dalke and Sime 1941 \\
\hline Home Range & $\begin{array}{l}3.2 \text { ha }\left(\delta^{*}\right) ; 2.1 \text { ha }(\$) \\
7.2 \text { ha }\left(\delta^{*}\right) ; 2.8 \text { ha }(\$) \\
7.8 \text { ha }\left(\delta^{*}\right) ; 2.4 \text { ha }(\$) \\
3.1 \text { ha }\left(\delta^{\prime}\right) ; 1.5 \text { ha }(\$)\end{array}$ & $\begin{array}{l}\text { winter - Pennsylvania } \\
\text { spring } \\
\text { summer } \\
\text { fall }\end{array}$ & Althoff and Storm 1989 \\
\hline Habitat Requirements & $\begin{array}{l}\text { wide variety of habitats } \\
\text { used; old fields } \\
\text { preferred }\end{array}$ & & Chapman et al. 1980 \\
\hline Population Density & $\begin{array}{l}0.41-2.10 / \mathrm{ha} \\
(\text { mean }=1.1 / \mathrm{ha})\end{array}$ & Michigan & Eberhardt et al. 1963 \\
\hline \multirow[t]{2}{*}{ Behavior } & $\begin{array}{l}\text { activity is greatest at } \\
\text { dusk and dawn } \\
\text { (crepuscular) }\end{array}$ & & Chapman et al. 1980 \\
\hline & $\begin{array}{l}\text { active year round - does } \\
\text { not hibernate }\end{array}$ & & EPA 1993a \\
\hline
\end{tabular}

'Suggested values for use in exposure assessment are in bold.

${ }^{b} \mathrm{~kg} / \mathrm{d}$ wet weight

${ }^{c} \mathrm{~kg} / \mathrm{d}$ dry weight 


\subsection{MINK (Mustela vison)}

\begin{tabular}{|c|c|c|c|}
\hline Parameter & Value $^{\mathrm{a}}$ & Comments & Reference \\
\hline \multirow[t]{2}{*}{ Body Weight } & $\begin{array}{l}0.9-1.6 \mathrm{~kg}(\delta) \\
0.7-1.1 \mathrm{~kg}(\$)\end{array}$ & & Linscombe et al. 1982 \\
\hline & $1.0 \mathrm{~kg}($ mean $\delta+q)$ & & $\begin{array}{l}\text { EPA } 1993 b \\
\text { Newell et al. } 1987\end{array}$ \\
\hline Food Consumption Rate ${ }^{b}$ & $0.137 \mathrm{~kg} / \mathrm{d}($ mean $\delta+q)$ & & $\begin{array}{l}\text { Bleavins and Aulerich } \\
1981\end{array}$ \\
\hline Water Consumption Rate & $0.099 \mathrm{~L} / \mathrm{d}$ & $\begin{array}{l}\text { estimated using } \\
\text { Equation } 18 ; \\
\text { assuming } 1.0 \mathrm{~kg} \mathrm{bw}\end{array}$ & \\
\hline Soil Consumption Rate ${ }^{c}$ & negligible & $\begin{array}{l}\text { sand observed in } 1.3 \% \\
\text { of mink scats; this } \\
\text { amount did not account } \\
\text { for any measurable scat } \\
\text { volume. }\end{array}$ & Hamilton 1940 \\
\hline Diet Composition & $\begin{array}{l}\text { Diverse diet includes: } \\
\text { mammals } \sim 46 \% \\
\text { fish } \sim 16 \% \\
\text { aquatic invertebrates } \\
\sim 15 \% \\
\text { amphibians } \sim 13 \% \\
\text { and birds } \sim 8 \%\end{array}$ & $\begin{array}{l}\text { Percentages represent } \\
\text { means of values from } \\
\text { four studies }\end{array}$ & $\begin{array}{l}\text { Hamilton } 1940 \text {, } \\
\text { Sealander 1943, } \\
\text { Korschgen 1958, } \\
\text { Burgess and Bider } 1980\end{array}$ \\
\hline \multirow[t]{3}{*}{ Home Range } & $\begin{array}{l}2.63 \mathrm{~km}(\delta) \\
1.85 \mathrm{~km}(q)\end{array}$ & stream - Sweden & Gerell 1970 \\
\hline & 770 ha $(\delta)$ & $\begin{array}{l}\text { prairie potholes, } \\
\text { Manitoba }\end{array}$ & Arnold and Fritzell 1987 \\
\hline & & $\begin{array}{l}\text { range size and shape } \\
\text { depends on habitat - } \\
\text { linear along streams, } \\
\text { circular in marshes }\end{array}$ & EPA 1993a \\
\hline Habitat Requirements & $\begin{array}{l}\text { aquatic habitats - } \\
\text { streams, lakes, marshes; }\end{array}$ & & $\begin{array}{l}\text { Burt and Grossenheider } \\
1976\end{array}$ \\
\hline Population Density & $0.03-0.085 / \mathrm{ha}$ & river - Montana & Mitchell 1961 \\
\hline Behavior & $\begin{array}{l}\text { nocturnal } \\
\text { active year-round, } \\
\text { does not hibernate }\end{array}$ & & EPA 1993a \\
\hline
\end{tabular}




\subsection{RED FOX (Vulpes vulpes)}

\begin{tabular}{|c|c|c|c|}
\hline Parameter & Value $^{a}$ & Comments & Reference \\
\hline \multirow[t]{3}{*}{ Body Weight } & $\begin{array}{l}5.25 \pm 0.18 \mathrm{~kg}(\delta) \\
4.13 \pm 0.11 \mathrm{~kg}(\%)\end{array}$ & Illinois & Storm et al. 1976 \\
\hline & $\begin{array}{l}4.82 \pm 0.081 \mathrm{~kg}(\delta) \\
3.94 \pm 0.079 \mathrm{~kg}(\$)\end{array}$ & lowa & \\
\hline & $4.5 \mathrm{~kg}$ & $\begin{array}{l}\text { mean } \delta+9 \text { for both } \\
\text { Illinois and Iowa }\end{array}$ & \\
\hline \multirow[t]{3}{*}{ Food Consumption Rate } & $0.596 \mathrm{~kg} / \mathrm{d}$ & see calculation below & $\begin{array}{l}\text { Vogtsberger and Barret } \\
1973\end{array}$ \\
\hline & $0.31 \mathrm{~kg} / \mathrm{d}$ & $\begin{array}{l}0.069 \mathrm{~g} / \mathrm{g} \mathrm{bw} / \mathrm{d} \text { for } \\
\text { nonbreeding adult times } \\
4.5 \mathrm{~kg} \mathrm{bw}\end{array}$ & Sargeant 1978 \\
\hline & $0.45 \mathrm{~kg} / \mathrm{d}$ & mean of both estimates & \\
\hline Water Consumption Rate & $0.38 \mathrm{~L} / \mathrm{d}$ & $\begin{array}{l}\text { estimated using } \\
\text { Equation } 18 ; \\
\text { assuming } 4.5 \mathrm{~kg} \mathrm{bw}\end{array}$ & \\
\hline \multirow[t]{2}{*}{ Soil Consumption Rate } & $2.8 \%$ & & Beyer et al. 1994 \\
\hline & $0.0126 \mathrm{~kg} / \mathrm{d}$ & $\begin{array}{l}\text { assuming diet of } 0.45 \\
\mathrm{~kg} / \mathrm{d}\end{array}$ & \\
\hline Diet Composition & $\begin{array}{l}\text { mammals }-68.8 \% \\
\text { birds }-12.0 \% \\
\text { plants }-10.4 \% \\
\text { insects }-0.9 \% \\
\text { misc. }-5.5 \%\end{array}$ & $\begin{array}{l}\text { Maryland, } \\
\text { Appalachian region }\end{array}$ & \\
\hline \multirow[t]{2}{*}{ Home Range } & $\begin{array}{l}699 \pm 137 \text { ha } \\
(9 \text { spring) }\end{array}$ & $\begin{array}{l}\text { Minnesota - forest, field, } \\
\text { swamp }\end{array}$ & Sargeant 1972 \\
\hline & $\begin{array}{l}717 \text { ha ( } \delta \text { all year) } \\
96 \text { ha ( } \$ \text { all year) }\end{array}$ & $\begin{array}{l}\text { Wisconsin - multiple } \\
\text { habitats }\end{array}$ & Ables 1969 \\
\hline \multirow[t]{2}{*}{ Habitat Requirements } & $\begin{array}{l}\text { wide and diverse - occur } \\
\text { in many habitats }\end{array}$ & & \\
\hline & $\begin{array}{l}\text { prefer mixture of forest } \\
\text { and open habitat }\end{array}$ & & $\begin{array}{l}\text { Burt and Grossenheider } \\
1976\end{array}$ \\
\hline Population Density & $0.046-0.077 / \mathrm{ha}$ & $\begin{array}{l}\text { "good fox range" in } \\
\text { North America }\end{array}$ & EPA 1993a \\
\hline Behavior & $\begin{array}{l}\text { active year round - does } \\
\text { not hibernate }\end{array}$ & & EPA 1993a \\
\hline
\end{tabular}

Suggested values for use in exposure assessment are in bold. 


\section{Red Fox (continued)}

b The following parameters were presented by Vogtsberger and Barret (1973):

food ingestion $=223 \mathrm{kcal} / \mathrm{kg} \mathrm{bw} / \mathrm{d}$

energy content of vertebrate food $=5.606 \mathrm{kcal} / \mathrm{g} \mathrm{dry} \mathrm{wt}$.

wet-dry weight conversion $\quad=1 \mathrm{~g}$ wet $\mathrm{wt}=0.3 \mathrm{~g}$ dry $\mathrm{wt}$

therefore:

$223 \mathrm{kcal} / \mathrm{kg} \mathrm{bw} / \mathrm{d} \times 4.5 \mathrm{~kg}$ bw $=1003.5 \mathrm{kcal} / \mathrm{d}$

$1003.5 \mathrm{kcal} / \mathrm{d} \times 1 \mathrm{~g} \mathrm{dry} \mathrm{wt.} / 5.606 \mathrm{kcal}=179 \mathrm{~g} \mathrm{dry} / \mathrm{d}$

$179 \mathrm{~g} \mathrm{dry} / \mathrm{d} \times 1 \mathrm{~g}$ wet $/ 0.3 \mathrm{~g}$ dry (wet-dry conversion) $=596 \mathrm{~g} / \mathrm{d}$

${ }^{\circ} \mathrm{kg} / \mathrm{d}$ wet weight

${ }^{\mathrm{d}} \mathrm{kg} / \mathrm{d}$ dry weight

\subsection{WHITE-TAILED DEER (Odocoileus virginianus)}

\begin{tabular}{|c|c|c|c|}
\hline Parameter & Value $^{\mathrm{a}}$ & Comments & Reference \\
\hline Body Weight & $\begin{array}{l}68 \mathrm{~kg}\left({ }^{\sharp)}\right. \\
45 \mathrm{~kg}(q) \\
56.5 \mathrm{~kg}\left(\text { mean } \delta^{+}+\right)\end{array}$ & & Smith 1991 \\
\hline Food Consumption Rate ${ }^{b}$ & $1.74 \mathrm{~kg} / \mathrm{d}$ & & Mautz et al. 1976 \\
\hline Water Consumption Rate & 3.7 L/d & $\begin{array}{l}\text { estimated using } \\
\text { Equation } 18 ; \\
\text { assuming } 56.5 \mathrm{~kg} \text { bw }\end{array}$ & \\
\hline Soil Consumption Rate ${ }^{c}$ & $\begin{array}{l}<2 \% \\
0.0348 \mathrm{~kg} / \mathrm{d}\end{array}$ & $\begin{array}{l}\text { assuming } 2 \% \text { soil and } \\
1.74 \mathrm{~kg} / \mathrm{d} \text { food } \\
\text { consumption rates }\end{array}$ & Beyer et al. 1994 \\
\hline
\end{tabular}

Diet Composition

exclusively herbivorous

diet diverse and variable,

Zim et al. 1951

depends on availability.

major foods:

- buds and twigs of

Smith 1991

trees and shrubs

- grasses and forbs

(summer)

- mast and fruits (fall)

Home Range

59 - 520 ha

Marchinton and Hirth

1984 
White-tailed Deer (continued)

\begin{tabular}{|c|c|c|c|}
\hline Parameter & Value $^{2}$ & Comments & Reference \\
\hline Habitat Requirements & $\begin{array}{l}\text { uses a wide variety of } \\
\text { habitats; favors forest- } \\
\text { field-farmland mosaic; } \\
\text { population density } \\
\text { directly related to } \\
\text { number and distribution } \\
\text { of forest openings }\end{array}$ & & Smith 1991 \\
\hline \multirow[t]{2}{*}{ Population Density } & $0.06 / \mathrm{ha}$ & $\begin{array}{l}\text { eastern mixed deciduous } \\
\text { forest - Tennessee }\end{array}$ & Barber 1984 \\
\hline & $0.39-0.78 / \mathrm{ha}$ & $\begin{array}{l}\text { oak-hickory forest - } \\
\text { midwest }\end{array}$ & $\begin{array}{l}\text { Torgerson and Porath } \\
1984\end{array}$ \\
\hline Behavior & $\begin{array}{l}\text { generally crepuscular } \\
\text { active year-round; does } \\
\text { not hibernate }\end{array}$ & & Smith 1991 \\
\hline
\end{tabular}

\subsection{AMERICAN ROBIN (Turdus migratorius)}

\begin{tabular}{|c|c|c|c|}
\hline Parameter & Value $^{2}$ & Comments & Reference \\
\hline Body Weight & $0.077 \mathrm{~kg}$ & & Dunning 1984 \\
\hline \multirow[t]{3}{*}{ Food Consumption Rate } & $\begin{array}{l}1.52 \mathrm{~g} / \mathrm{g} \text { bw/d or } \\
117 \mathrm{~g} / \mathrm{d}\end{array}$ & assuming a $0.077 \mathrm{bw}$ & Hazelton et al. 1984 \\
\hline & $\begin{array}{l}0.89 \mathrm{~g} / \mathrm{g} \text { bw } / \mathrm{d} \text { or } \\
68.5 \mathrm{~g} / \mathrm{d}\end{array}$ & assuming a $0.077 \mathrm{bw}$ & $\begin{array}{l}\text { Skorupa and Hothem } \\
1985\end{array}$ \\
\hline & $0.093 \mathrm{~kg} / \mathrm{d}$ & mean of both values & \\
\hline Water Consumption Rate & $0.0106 \mathrm{~L} / \mathrm{d}$ & $\begin{array}{l}\text { estimated using } \\
\text { Equation } 19 ; \\
\text { assuming } 0.077 \mathrm{~kg} \text { bw }\end{array}$ & \\
\hline
\end{tabular}


American Robin (continued)

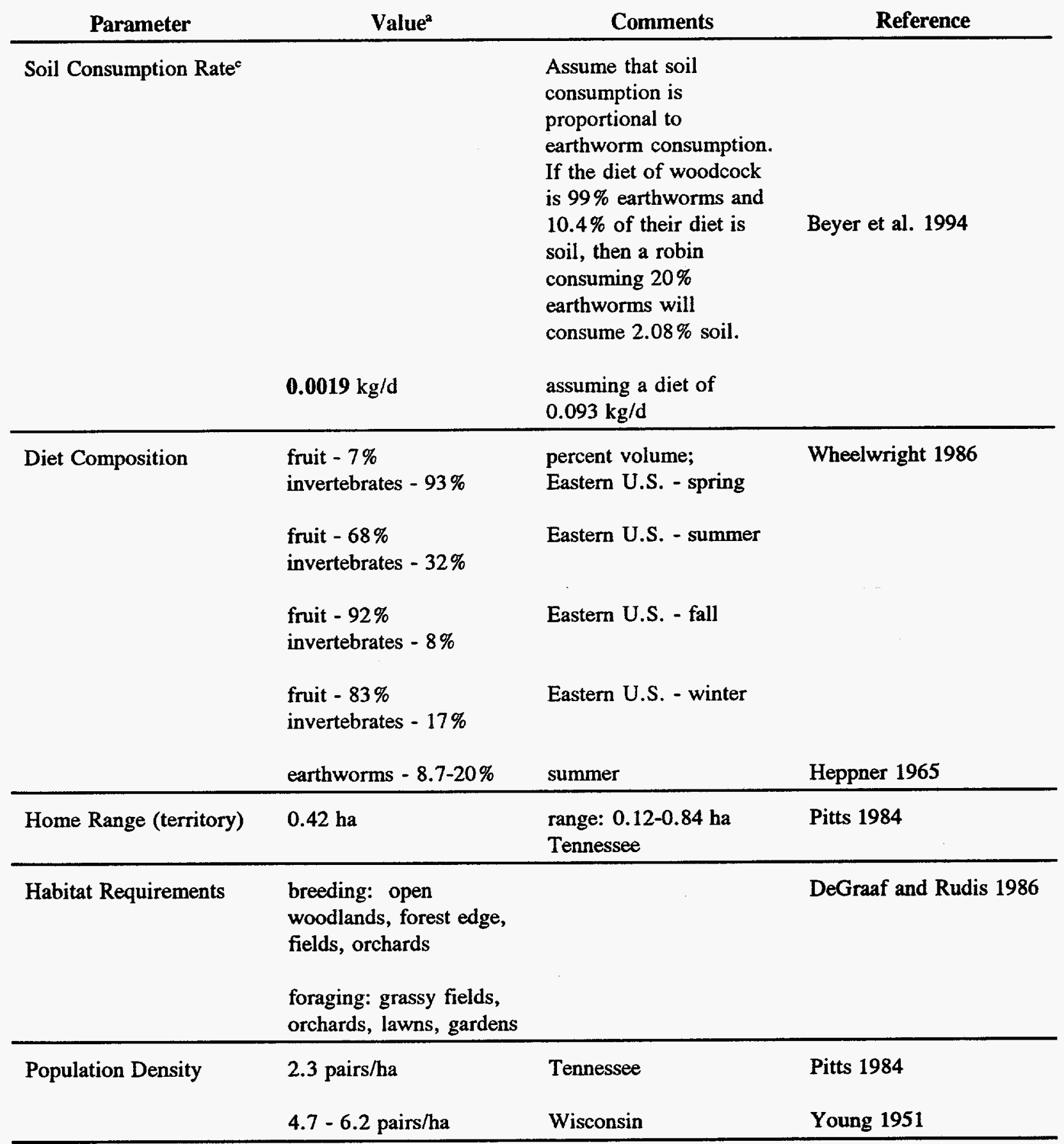


American Robin (continued)

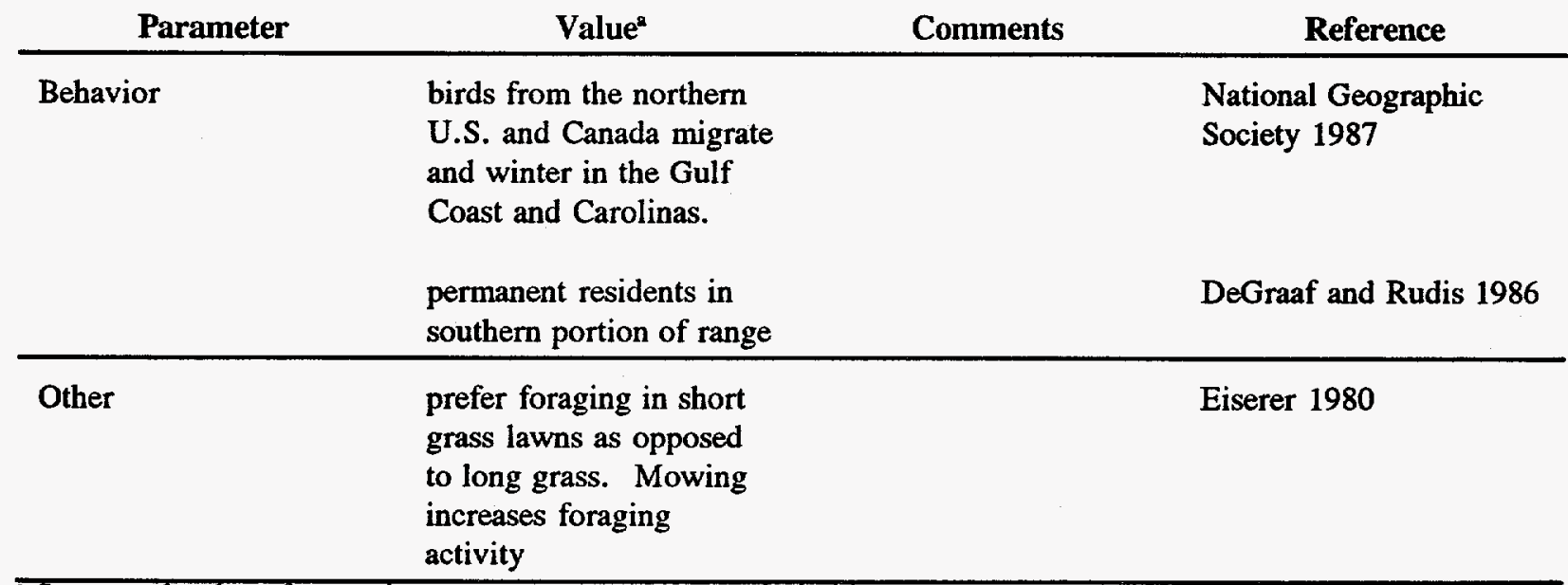

Suggested values for use in exposure assessment are in bold.

${ }^{b} \mathrm{~kg} / \mathrm{d}$ wet weight

${ }^{c} \mathrm{~kg} / \mathrm{d}$ dry weight

\subsection{AMERICAN WOODCOCK (Scolopax minor)}

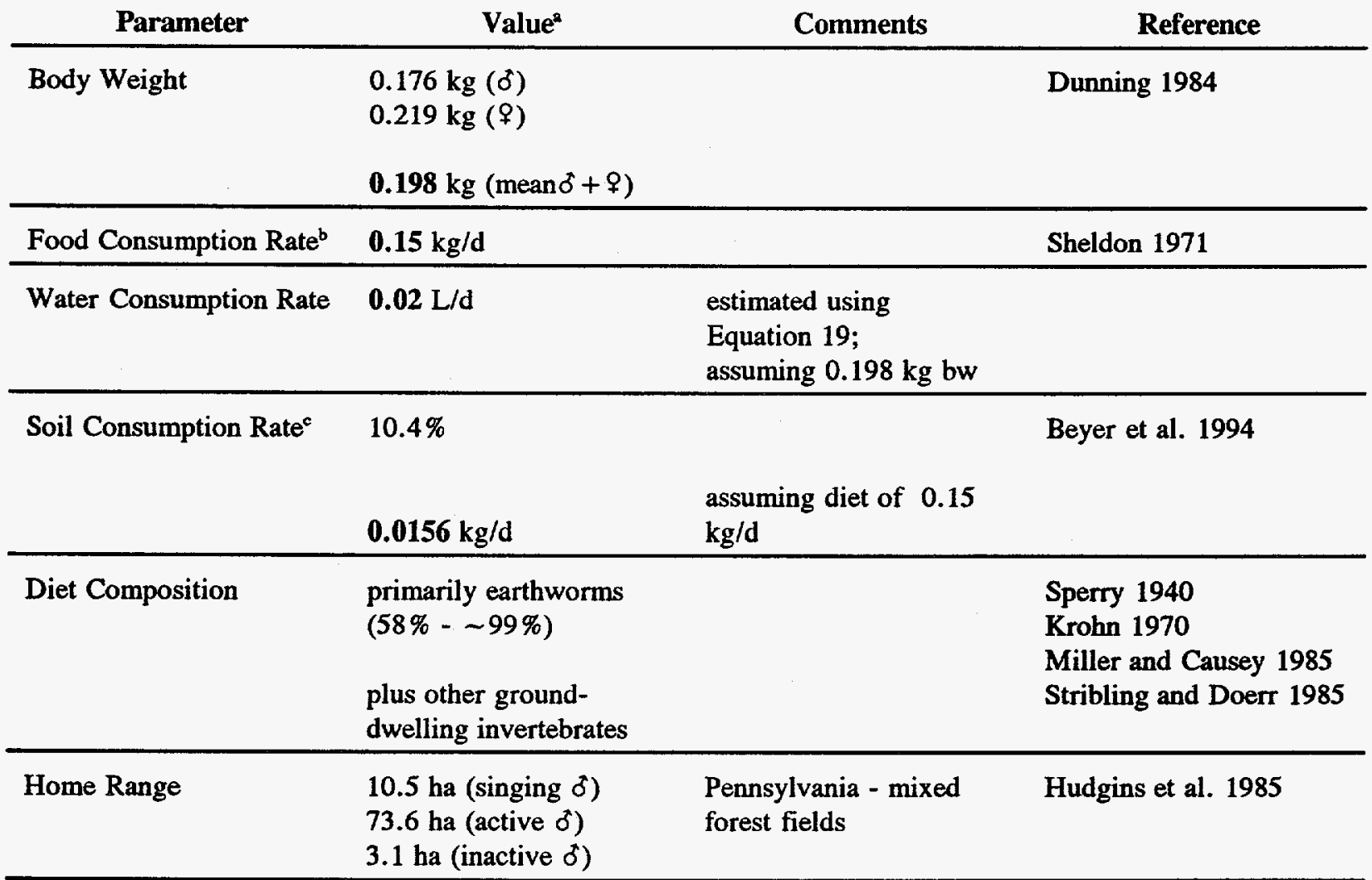


American Woodcock (continued)

\begin{tabular}{|c|c|c|c|}
\hline Parameter & Value $^{2}$ & Comments & Reference \\
\hline Habitat Requirements & $\begin{array}{l}\text { breeding: moist early } \\
\text { successional woodlands, } \\
\text { swamps, river bottoms, } \\
\text { alder thickets }\end{array}$ & & DeGraaf and Rudis 1986 \\
\hline & $\begin{array}{l}\text { feeding: moist open } \\
\text { pasture, cultivated fields, } \\
\text { stream banks }\end{array}$ & & \\
\hline \multirow[t]{2}{*}{ Population Density } & $\begin{array}{l}3.4 \text { /ha } \\
0.2 / \text { ha } \\
0.034 \text { /ha }\end{array}$ & $\begin{array}{l}\text { winter-North Carolina } \\
\text { untilled soy stubble } \\
\text { untilled corn stubble } \\
\text { rebedded corn }\end{array}$ & Connors and Doerr 1982 \\
\hline & 0.21 nests $/$ ha & $\begin{array}{l}\text { mixed pine hardwoods- } \\
\text { Pennsylvania }\end{array}$ & Coon et al. 1982 \\
\hline Behavior & $\begin{array}{l}\text { migrate from northern } \\
\text { breeding range to } \\
\text { wintering range in south } \\
\text { Atlantic and gulf coast } \\
\text { states } \\
\text { early migrants; leave } \\
\text { wintering grounds in } \\
\text { February, arrive at } \\
\text { northern breeding } \\
\text { grounds late March }\end{array}$ & & Sheldon 1971 \\
\hline
\end{tabular}

Suggested values for use in exposure assessment are in bold.

${ }^{b} \mathrm{~kg} / \mathrm{d}$ wet weight

${ }^{c} \mathrm{~kg} / \mathrm{d}$ dry weight

4.11 WILD TURKEY (Meleagris gallopavo)

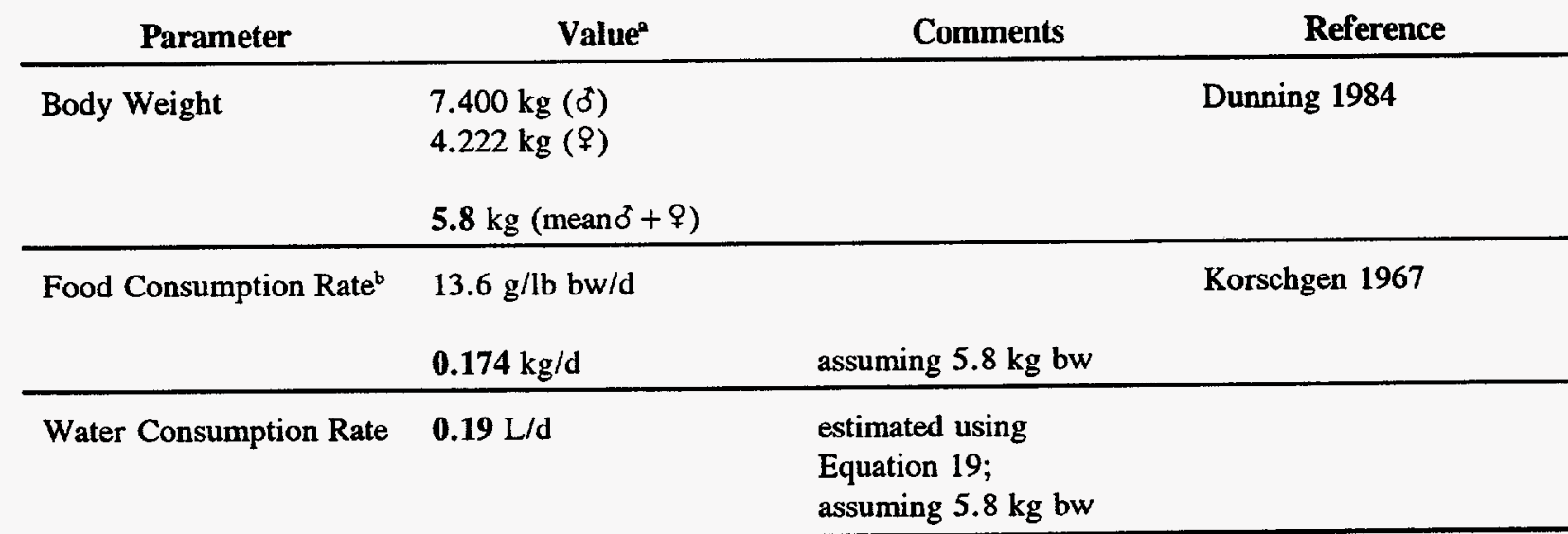


Wild Turkey (continued)

\begin{tabular}{|c|c|c|c|}
\hline Parameter & Value $^{a}$ & Comments & Reference \\
\hline \multirow[t]{2}{*}{ Soil Consumption Rate ${ }^{c}$} & $9.3 \%$ & & Beyer et al. 1994 \\
\hline & $0.0162 \mathrm{~kg} / \mathrm{d}$ & $\begin{array}{l}\text { assuming } 0.174 \mathrm{~kg} / \mathrm{d} \\
\text { food consumption rates }\end{array}$ & \\
\hline \multirow[t]{2}{*}{ Diet Composition } & $\begin{array}{l}\text { plant material (mast, } \\
\text { fruit, seeds, some } \\
\text { foliage) }-90.3 \%\end{array}$ & & Korschgen 1967 \\
\hline & $\begin{array}{l}\text { animal material (insects, } \\
\text { crayfish, snails, } \\
\text { salamanders) }-9.7 \%\end{array}$ & & \\
\hline Home Range & $150-190$ ha & & DeGraaf and Rudis 1986 \\
\hline Habitat Requirements & $\begin{array}{l}\text { mast-producing } \\
\text { woodlands with } \\
\text { associated fields and } \\
\text { abundant water }\end{array}$ & & DeGraaf and Rudis 1986 \\
\hline \multirow[t]{2}{*}{ Population Density } & 0.03 tha & West Virginia & DeGraaf and Rudis 1986 \\
\hline & $0.06-0.076 / \mathrm{ha}$ & in 'ideal' habitat & DeGraaf and Rudis 1986 \\
\hline \multirow[t]{3}{*}{ Behavior } & $\begin{array}{l}\text { forage primarily on the } \\
\text { ground }\end{array}$ & & $\begin{array}{l}\text { National Geographic } \\
\text { Society } 1987\end{array}$ \\
\hline & roost in trees at night & & \\
\hline & $\begin{array}{l}\text { year-round resident; does } \\
\text { not migrate }\end{array}$ & & \\
\hline
\end{tabular}

'Suggested values for use in exposure assessment are in bold.

${ }^{b} \mathrm{~kg} / \mathrm{d}$ wet weight

${ }^{\circ} \mathrm{kg} / \mathrm{d}$ dry weight

\subsection{BELTED KINGFISHER (Ceryle alcyon)}

\begin{tabular}{|c|c|c|c|}
\hline Parameter & Value $^{2}$ & Comments & Reference \\
\hline Body Weight & $0.148 \mathrm{~kg}$ & & Dunning 1984 \\
\hline \multirow[t]{2}{*}{ Food Consumption Rate } & $50 \%$ bw & & Alexander 1977 \\
\hline & $0.075 \mathrm{~kg} / \mathrm{d}$ & assuming $0.148 \mathrm{~kg}$ bw & \\
\hline
\end{tabular}


Belted Kingfisher (continued)

\begin{tabular}{|c|c|c|c|}
\hline Parameter & Value $^{2}$ & Comments & Reference \\
\hline Water Consumption Rate & $0.016 \mathrm{~L} / \mathrm{d}$ & $\begin{array}{l}\text { estimated using } \\
\text { Equation } 19 ; \\
\text { assuming } 0.148 \mathrm{~kg} \mathrm{bw}\end{array}$ & \\
\hline
\end{tabular}

\begin{tabular}{llll}
\hline Soil Consumption Rate & \multicolumn{2}{c}{$\begin{array}{l}\text { as a piscivore, assumed } \\
\text { to be negligible }\end{array}$} & Davis 1982 \\
\hline Diet Composition & $\begin{array}{l}\text { Cyprinids }-76.4 \% \\
\text { other fish }-10.2 \% \\
\text { crayfish }-13.3 \%\end{array}$ & creek-Ohio & Landrum et al. 1993 \\
& $\begin{array}{l}\text { lizards, small snakes, } \\
\text { frogs, salamanders, and } \\
\text { insects may be consumed } \\
\text { if fish are unavailable }\end{array}$ & \\
\hline Home Range & $\begin{array}{l}1.03 \mathrm{~km} \text { (breeding) } \\
0.39 \mathrm{~km} \text { (non-breeding) }\end{array}$ & creek-Ohio & Davis 1982 \\
& $2.19 \mathrm{~km}$ (breeding) & $\begin{array}{l}\text { stream summer- } \\
\text { Pennsylvania }\end{array}$ & Brooks and Davis 1987 \\
\hline
\end{tabular}

\begin{tabular}{lll}
\hline Habitat Requirements & $\begin{array}{l}\text { use diverse aquatic } \\
\text { habitats (stream, river, } \\
\text { lake, marsh, coastline) }\end{array}$ & Brooks and Davis 1987 \\
& $\begin{array}{l}\text { require high vertical } \\
\text { banks composed of } \\
>75 \% \text { sand and }<7 \% \\
\text { clay for nest construction }\end{array}$ & \\
& $\begin{array}{l}\text { prefer relatively clear } \\
\text { waters free of thick } \\
\text { vegetation }\end{array}$ & Bent 1940 \\
\hline Population Density & $\begin{array}{l}\text { 0.11 - 0.19 pairs/km } \\
\text { shore }\end{array}$ & Brooks and Davis 1987 \\
\hline Behavior & $\begin{array}{l}\text { while most migrate from summer- } \\
\text { northern parts of range, } \\
\text { some may stay in areas } \\
\text { where water remains ice- } \\
\text { free }\end{array}$ & Bent 1940 \\
\hline
\end{tabular}

'Suggested values for use in exposure assessment are in bold.

${ }^{b} \mathrm{~kg} / \mathrm{d}$ wet weight

${ }^{\circ} \mathrm{kg} / \mathrm{d}$ dry weight 


\subsection{GREAT BLUE HERON (Ardea herodias)}

\begin{tabular}{|c|c|c|c|}
\hline Parameter & Value & Comments & Reference \\
\hline Body Weight & $\begin{array}{l}2.576 \mathrm{~kg} \mathrm{( \delta )} \\
2.204 \mathrm{~kg} \mathrm{(१)} \\
2.39 \mathrm{~kg}(\text { mean } \delta+q)\end{array}$ & & Dunning 1984 \\
\hline Food Consumption Rate ${ }^{b}$ & $0.42 \mathrm{~kg} / \mathrm{d}$ & $\begin{array}{l}\text { estimated using } \\
\text { allometric equation } \\
\text { specific for herons and } \\
\text { egrets } \\
\text { assuming } 5.8 \mathrm{~kg} \mathrm{bw}\end{array}$ & Kushlan 1978 \\
\hline Water Consumption Rate & $0.1058 \mathrm{~L} / \mathrm{d}$ & $\begin{array}{l}\text { estimated using } \\
\text { Equation } 19 ; \\
\text { assuming } 2.39 \mathrm{~kg} \mathrm{bw}\end{array}$ & \\
\hline
\end{tabular}

$\begin{array}{ll}\text { Soil Consumption Rate } & \begin{array}{l}\text { as a piscivore, assumed } \\ \text { to be negligible }\end{array}\end{array}$

\begin{tabular}{|c|c|c|c|}
\hline Diet Composition & $\begin{array}{l}\text { diet predominantly fish } \\
\text { but may include } \\
\text { crustaceans, insects, } \\
\text { snails, amphibians, } \\
\text { reptiles, birds, and } \\
\text { mammals }\end{array}$ & & $\begin{array}{l}\text { Kushlan } 1978 \\
\text { Collazo } 1985 \\
\text { Hoffman } 1978\end{array}$ \\
\hline \multirow[t]{2}{*}{$\begin{array}{l}\text { Home Range } \\
\text { (foraging distance from } \\
\text { colony) }\end{array}$} & $3.1 \mathrm{~km}$ & $\begin{array}{l}\text { up to } 24.2 \mathrm{~km}- \\
\text { river-S. Dakota }\end{array}$ & EPA 1993a \\
\hline & $7-8 \mathrm{~km}$ & coastal-N. Carolina & Short and Cooper 1985 \\
\hline \multirow[t]{3}{*}{ Habitat Requirements } & $\begin{array}{l}\text { use both coastal and } \\
\text { inland water-associated } \\
\text { habitats }\end{array}$ & & Short and Cooper 1985 \\
\hline & $\begin{array}{l}\text { foraging: shallow shores } \\
\text { of ponds, lakes, streams, } \\
\text { wet meadows, wooded } \\
\text { swamps, bays, and } \\
\text { marshes }\end{array}$ & & DeGraaf and Rudis 1986 \\
\hline & $\begin{array}{l}\text { breeding: trees for } \\
\text { rookery sites. In } \\
\text { absence of trees will use } \\
\text { rock ledges, cliffs, and } \\
\text { artificial structures }\end{array}$ & & Short and Cooper 1985 \\
\hline
\end{tabular}




\section{Great Blue Heron (continued)}

\begin{tabular}{llll}
\multicolumn{1}{c}{ Parameter } & \multicolumn{1}{c}{ Value } & Comments & Reference \\
\hline Population Density & $\begin{array}{l}\text { nest colonially; } \\
\text { therefore, population } \\
\text { density depends on } \\
\text { availability of nest } \\
\text { habitat and suitable } \\
\text { foraging habitat }\end{array}$ & EPA 1993a \\
& $\begin{array}{l}\text { may or may not defend a } \\
\text { feeding territory } \\
\text { depending on local } \\
\text { population size and food } \\
\text { availability }\end{array}$ & Kushlan 1978 \\
\hline Behavior & $\begin{array}{l}\text { Migrates in northern } \\
\text { U.S. and southern } \\
\text { Canada; year round } \\
\text { resident from WV, PA } \\
\text { south. }\end{array}$ & \\
\hline
\end{tabular}

${ }^{2}$ Suggested values for use in exposure assessment are in bold.

b Allometric equation for estimation of food consumption by herons and egrets is:

$$
\log F I R=0.966 \log b w-0.640
$$

where:

FIR $=$ food ingestion rate ( $\mathrm{g} / \mathrm{d}$ wet weight)

bw $=$ body weight $(\mathrm{g})$

${ }^{\mathrm{c}} \mathrm{kg} / \mathrm{d}$ wet weight

${ }^{\mathrm{d}} \mathrm{kg} / \mathrm{d}$ dry weight

\subsection{BARN OWL (Tyto alba)}

\begin{tabular}{|c|c|c|c|}
\hline Parameter & Value $^{\mathbf{2}}$ & Comments & Reference \\
\hline Body Weight & $\begin{array}{l}0.442 \mathrm{~kg}\left({ }^{\ddagger}\right) \\
0.490 \mathrm{~kg}(\$) \\
0.466 \mathrm{~kg}\left(\text { mean }{ }^{\dagger}+\$\right)\end{array}$ & & Dunning 1984 \\
\hline Food Consumption Rate & $\begin{array}{l}0.1-0.15 \mathrm{~kg} / \mathrm{d} \\
0.125 \mathrm{~kg} / \mathrm{d} \text { (mean) }\end{array}$ & & Johnsgard 1988 \\
\hline Water Consumption Rate & $0.035 \mathrm{~L} / \mathrm{d}$ & $\begin{array}{l}\text { estimated using } \\
\text { Equation } 19 ; \\
\text { assuming } 0.466 \mathrm{~kg} \mathrm{bw}\end{array}$ & \\
\hline
\end{tabular}


Barn Owl (continued)

\begin{tabular}{|c|c|c|c|}
\hline Parameter & Value $^{2}$ & Comments & Reference \\
\hline Soil Consumption Rate ${ }^{\mathfrak{c}}$ & $\begin{array}{l}\text { while some soil attached } \\
\text { to prey may be ingested, } \\
\text { amount is assumed to } \\
\text { negligible }\end{array}$ & & \\
\hline \multirow[t]{2}{*}{ Diet Composition } & $\begin{array}{l}\text { predominantly small } \\
\text { mammals (Microtus, } \\
\text { Blarina, Peromyscus, } \\
\text { etc.) } 80-90 \% \text {; } \\
\text { occasionally birds, } \\
\text { amphibians, reptiles, and } \\
\text { insects. }\end{array}$ & & $\begin{array}{l}\text { Johnsgard } 1988 \\
\text { DeGraaf and Rudis } 1986\end{array}$ \\
\hline & $\begin{array}{l}\text { mean prey size: } \\
27-123 \mathrm{~g}\end{array}$ & & $\begin{array}{l}\text { Knight and Jackman } \\
1984\end{array}$ \\
\hline Home Range & $\sim 250 \mathrm{ha}$ & & Johnsgard 1988 \\
\hline Habitat Requirements & $\begin{array}{l}\text { feeding: marshes, } \\
\text { meadows, fields, } \\
\text { barnyards, brushy areas } \\
\text { that attract rodents. } \\
\text { Generally avoids } \\
\text { woodlands. } \\
\text { breeding: old buildings, } \\
\text { trees, cliffs, quarries. }\end{array}$ & & DeGraaf and Rudis 1986 \\
\hline Population Density & uncommon to rare & & DeGraaf and Rudis 1986 \\
\hline \multirow[t]{2}{*}{ Behavior } & $\begin{array}{l}\text { nocturnal, roosts during } \\
\text { day, hunts at night. }\end{array}$ & & DeGraaf and Rudis 1986 \\
\hline & $\begin{array}{l}\text { except for extreme } \\
\text { northern range, does not } \\
\text { migrate }\end{array}$ & & $\begin{array}{l}\text { National Geographic } \\
\text { Society } 1987\end{array}$ \\
\hline
\end{tabular}

\subsection{RED-TAILED HAWK (Buteo jamaicensis)}

\begin{tabular}{llll}
\multicolumn{1}{c}{ Parameter } & \multicolumn{1}{c}{ Value $^{2}$} & Comments & Reference \\
\hline Body Weight & $1.028 \mathrm{~kg}(\delta)$ & & Dunning 1984 \\
& $1.224 \mathrm{~kg}(\$)$ & \\
& $1.126 \mathrm{~kg}\left(\right.$ mean $\left.{ }^{\jmath}+9\right)$ & \\
\hline
\end{tabular}


Red-tailed Hawk (continued)

\begin{tabular}{|c|c|c|c|}
\hline Parameter & Value $^{2}$ & Comments & Reference \\
\hline Food Consumption Rate ${ }^{b}$ & $\begin{array}{l}0.117 \mathrm{~kg} / \mathrm{d} \\
0.136 \mathrm{~kg} / \mathrm{d} \\
0.073 \mathrm{~kg} / \mathrm{d} \\
\\
0.109 \mathrm{~kg} / \mathrm{d} \\
\end{array}$ & $\begin{array}{l}\text { ( } \delta, \text { fall/winter) } \\
(\$, \text { fall/winter) } \\
(\delta, \text { spring/summer) } \\
\text { (mean } \$ \text { and } \delta, \text { all year) }\end{array}$ & $\begin{array}{l}\text { Craighead and Craighead } \\
1969\end{array}$ \\
\hline Water Consumption Rate & $0.064 \mathrm{~L} / \mathrm{d}$ & $\begin{array}{l}\text { estimated using } \\
\text { Equation } 19 ; \\
\text { assuming } 1.126 \mathrm{~kg} \mathrm{bw}\end{array}$ & \\
\hline Soil Consumption Rate & $\begin{array}{l}\text { while some soil attached } \\
\text { to prey may be ingested, } \\
\text { amount is assumed } \\
\text { negligible }\end{array}$ & & \\
\hline \multirow[t]{3}{*}{ Diet Composition } & $\begin{array}{l}\text { predominantly small } \\
\text { mammals }\end{array}$ & & DeGraaf and Rudis 1986 \\
\hline & $\begin{array}{l}\text { small mammal }-78.5 \% \\
\text { bird }-8.5 \% \\
\text { snake }-13.0 \%\end{array}$ & $\begin{array}{l}\text { Oregon - pasture and } \\
\text { wheat fields }\end{array}$ & Janes 1984 \\
\hline & $\begin{array}{l}\text { mammals: } 13.2-18.7 \% \\
\text { birds: } 78.6-80.1 \%\end{array}$ & $\begin{array}{l}\text { Montana, during nesting } \\
\text { period }\end{array}$ & $\begin{array}{l}\text { Craighead and Craighead } \\
1969\end{array}$ \\
\hline \multirow[t]{2}{*}{ Home Range } & 233 ha & $\begin{array}{l}\text { pasture and wheat } \\
\text { fields-Oregon }\end{array}$ & Janes 1984 \\
\hline & $\begin{array}{l}1936 \text { ha } \\
\text { ( } 957-2465 \text { ha range) }\end{array}$ & $\begin{array}{l}\text { prairie-pinyon/juniper } \\
\text { woodland-Colorado; } \\
\text { mean of } 4 \text { birds; } 95 \% \\
\text { ellipse and systematic } \\
\text { relocation }\end{array}$ & $\begin{array}{l}\text { Andersen and Rongstad } \\
1989\end{array}$ \\
\hline
\end{tabular}

\begin{tabular}{lll}
\hline Habitat Requirements & $\begin{array}{l}\text { use wide range of } \\
\text { habitats, prefer } \\
\text { landscapes containing } \\
\text { mixture of old fields, } \\
\text { wetlands, and pasture for } \\
\text { foraging interspersed } \\
\text { with trees for perching } \\
\text { and nesting }\end{array}$ & DeGraaf and Rudis 1986 \\
&
\end{tabular}

\begin{tabular}{lll}
\hline Population Density & $0.03->0.005$ pairs $/$ ha & EPA 1993a \\
\hline
\end{tabular}


Red-tailed Hawk (continued)

\begin{tabular}{|c|c|c|c|}
\hline Parameter & Value $^{\mathbf{a}}$ & Comments & Reference \\
\hline Behavior & $\begin{array}{l}\text { territorial throughout } \\
\text { year }\end{array}$ & & EPA 1993a \\
\hline & $\begin{array}{l}\text { northerly populations } \\
\text { migrate; those in the } \\
\text { south do not }\end{array}$ & & $\begin{array}{l}\text { National Geographic } \\
\text { Society } 1987\end{array}$ \\
\hline
\end{tabular}

\section{APPLICATION OF THE MODELS}

To provide an example of the application of the models described in Sects. 2 and 3, contaminant exposure to wildlife was estimated using data from the Filled Coal Ash Pond (FCAP), a source operable unit on the ORR. Exposure was estimated for the meadow vole, red fox, white-tailed deer, and red-tailed hawk and compared to toxicological benchmarks obtained from Opresko et al. (1994).

\subsection{SITE DESCRIPTION}

The FCAP is a flat, 3.6-ha area located in the McCoy Branch watershed on the south slope of Chestnut Ridge, $0.8 \mathrm{~km}$ south of the Y-12 Plant. The site was constructed by building a 19-m high earthen dam across Upper McCoy Branch. The FCAP was built in 1955 to serve as a settling basin for coal ash from the Y-12 steam plant and continued to receive ash until 1989. Approximately 1-18 $\mathrm{m}$ of ash has accumulated behind the dam.

Vegetation has become established on the FCAP and grows directly in the coal ash. The difference in water level in the ash has resulted in two distinct vegetative zones. The lower one-third of the area is vegetated by hardwood trees with a dense understory. The upper portion of the FCAP has a higher water table and is vegetated by willows, sedges, and grasses. Small wetland areas with associated ponds occur in the northeast and northwest corners of the FCAP. Fish do not dwell in the ponds. Field observations, in addition to comparison of habitat requirements to habitat available on the FCAP, suggest that meadow vole, red fox, white-tailed deer, and red-tailed hawk make use of the site.

\subsection{DATA}

The following data were available to estimate contaminant exposure to wildlife: concentrations of arsenic, cadmium, chromium, lead, mercury, selenium, and thallium in coal ash, surface water, small mammals, and vegetation from the FCAP. In this example, actual measured values of contaminant residues were available for both abiotic media (ash and water) and some biota (plants and small mammals). In many instances, however, available data are likely to be 
restricted to contaminant concentrations in abiotic media. This frequently occurs with screeninglevel ecological risk assessments because they rely on existing data (which often consists only of concentrations in abiotic media).

Because contaminant concentrations in plant and animal wildlife foods are needed to fully estimate contaminant exposure for wildlife, if these data are not available, they must be generated from existing abiotic data. This may be done by using uptake factors that describe the relationship between contaminant concentrations in soil or water and that in biota. By multiplying the contaminant concentration in abiotic media by the appropriate uptake factor, concentrations in biota may be estimated.

Soil-plant uptake factors, for both reproductive and vegetative plant parts, were developed for inorganic contaminants (e.g., metals, radionuclides, etc.) by Baes et al. (1984). Travis and Arms (1988) report that uptake factors for organic chemicals in vegetation are inversely proportional to the square-root of the octanol-water partitioning coefficient $\left(\mathrm{K}_{\mathrm{ow}}\right)$. Uptake of organics by earthworms may be estimated according to the method of Menzie et al. (1992) where:

$$
\mathrm{UF}=\left(\mathrm{Y}_{\mathrm{L}}\right) /\left(0.66 * \mathrm{f}_{\mathrm{oc}}\right)
$$

where:

$\mathrm{UF}=$ uptake factor

$\mathrm{Y}_{\mathrm{L}} \quad=$ earthworm lipid content

$f_{o c} \quad=$ organic carbon content of soil

Gish and Hughes (1982) report that the mean lipid content of earthworms is $0.84 \%$. However, soil organic carbon content must be measured on a site-by-site basis. Values to estimate uptake of metals by earthworms may be obtained from the published literature such as Beyer and Stafford (1993).

After data have been obtained, a decision must be made as to which value to use to estimate exposure. Exposure may be estimated using several values to represent contaminant concentrations in media: the mean, upper $95 \%$ confidence interval on the mean (95\% UCL), or the maximum value. To be conservative, risk assessments generally use either the $95 \%$ UCL or the maximum value. For sedentary species (e.g., plants, benthic invertebrates) that will be exposed at only one location, the maximum value is the most appropriate value to use. Because wildlife are mobile, they may be exposed to contamination from multiple locations within a site. Therefore, the most appropriate value to use for wildlife is the 95\% UCL.

\subsection{EXPOSURE ESTIMATION AND EVALUATION}

Exposure estimates were calculated using data from the FCAP. Assumptions used in the estimates are outlined and the significance of the results are evaluated in the following text.

\subsubsection{Meadow Vole}

Estimates of contaminant exposure experienced by meadow voles were made using Equation 
4 and the following assumptions.

1) body weight $=0.044 \mathrm{~kg}$

2) ash consumption rate $=$ soil consumption rate $=0.00012 \mathrm{~kg} / \mathrm{d}$

3) water consumption rate $=0.006 \mathrm{~L} / \mathrm{d}$

4) food consumption rate $=0.005 \mathrm{~kg} / \mathrm{d}$

5) contaminant concentrations measured in vegetation from the FCAP is representative of that in plants (and plant parts) consumed by meadow voles

6) because the home range of voles $(0.037-0.083 \mathrm{ha})$ is smaller than the size of the FCAP ( $3.6 \mathrm{ha}$ ), $100 \%$ of food, water, and ash consumed was obtained from the FCAP.

Table 2 presents contaminant concentrations and exposure estimates for the meadow vole. Contaminant concentrations in ash are in $\mathrm{mg} / \mathrm{kg}$ dry weight while that in food is in $\mathrm{mg} / \mathrm{kg}$ wet weight.

To determine which contaminants are at potential hazardous concentrations, total exposure estimates should be compared to no observed adverse effects levels (NOAELs) for each contaminant. Estimated exposures to arsenic and selenium exceed NOAELs (Table 2); therefore, these contaminants may be adversely affecting meadow voles at the FCAP.

Contribution of each medium to total contaminant exposure helps to direct remedial actions and may be determined by dividing total exposure by the exposure estimate for each medium. For meadow voles on the FCAP, with the exception of arsenic, $70 \%$ to $100 \%$ of contaminant exposure is from food, and $0 \%$ to $30 \%$ is from incidental ash ingestion (Table 2). Water accounted for less than $1 \%$ of exposure. For arsenic, ash contributed $64 \%$ and food $36 \%$ of total exposure.

\subsubsection{Red Fox}

Estimates of contaminant exposure experienced by red fox were made using Equations 5, 6, and 7 and the following assumptions.

1) body weight $=4.5 \mathrm{~kg}$

2) ash consumption rate $=$ soil consumption rate $=0.0126 \mathrm{~kg} / \mathrm{d}$

3) water consumption rate $=0.38 \mathrm{~L} / \mathrm{d}$

4) food consumption rate $=0.45 \mathrm{~kg} / \mathrm{d}$

5) proportion of plant material in the diet $=0.104$

6) contaminant concentrations measured in vegetation from the FCAP are representative of that in plants (and plant parts) consumed by red fox

7) proportion of mammals in the diet $=0.688$

8) contaminant concentrations measured in small mammals from the FCAP are representative of that in small mammals consumed by red fox

9) no other foods consumed by red fox are contaminated

10) home range size for red fox $=96$ ha

11) use of the FCAP and therefore consumption of food, water, or ash from the FCAP is proportional to the size of the FCAP ( $3.6 \mathrm{ha}$ ) in relation to the home range size for red fox (96 ha) or 0.0375 ha 
Table 3 presents contaminant concentrations and exposure estimates for red fox. Contaminant concentrations in ash are in $\mathrm{mg} / \mathrm{kg}$ dry weight while that in food is in $\mathrm{mg} / \mathrm{kg}$ wet weight.

Comparison of the total exposure estimates to NOAELs for each contaminant indicate that exposure to selenium exceeds the NOAEL. Medium contributions to total contaminant exposure were similar to that for meadow voles; except for arsenic, most exposure was from food, followed by ash (Table 4). Water contributed little exposure.

\subsubsection{White-tailed Deer}

Estimates of contaminant exposure experienced by white-tailed deer were made using Equation 7 and the following assumptions.

1) body weight $=56.5 \mathrm{~kg}$

2) ash consumption rate $=$ soil consumption rate $=0.0348 \mathrm{~kg} / \mathrm{d}$

3) water consumption rate $=3.7 \mathrm{~L} / \mathrm{d}$

4) food consumption rate $=1.74 \mathrm{~kg} / \mathrm{d}$

5) contaminant concentrations measured in vegetation from the FCAP are representative of that in plants (and plant parts) consumed by white-tailed deer

6) to be conservative, the smallest reported home range was used. Therefore, home range size for white-tailed deer $=59$ ha

7) use of the FCAP and therefore consumption of food, water, or ash from the FCAP is proportional to the size of the FCAP ( $3.6 \mathrm{ha})$ in relation to the home range size for whitetailed deer ( $59 \mathrm{ha})$ or 0.061

Table 5 presents contaminant concentrations and exposure estimates for white-tailed deer. Contaminant concentrations in ash are in $\mathrm{mg} / \mathrm{kg}$ dry weight while that in food is in $\mathrm{mg} / \mathrm{kg}$ wet weight.

Comparison of the total exposure estimates to NOAELs for each contaminant indicate that only selenium exceeds its NOAEL and almost all exposure $(\sim 98.8 \%)$ is attributable to plant consumption (Table 5).

In addition to being exposed to contaminants in ash through incidental consumption, whitetailed deer may be exposed by consuming ash to meet mineral needs. As discussed previously, because most plant material contains little sodium (Robbins 1993), the diet of many herbivores, including white-tailed deer, is often sodium deficient. To compensate for this sodium deficiency, many ungulates locate and consume soils rich in sodium (Weeks 1978). Coal ash on the FCAP is rich in sodium relative to background soils, and areas where white-tailed deer appear to have consumed ash have been observed at the FCAP.

Pletscher (1987) estimates that white-tailed deer require $301 \mathrm{mg} \mathrm{Na} / \mathrm{d}$ of which only $23 \mathrm{mg}$ $\mathrm{Na} / \mathrm{d}$ is provided in their diet. This leaves a sodium deficit of $278 \mathrm{mg} \mathrm{Na} / \mathrm{d}$ to be made up from other sources. An exposure estimate was calculated in which it was estimated that a deer would consume the amount of ash sufficient to eliminate the sodium deficit. To estimate the amount of ash consumed, it was assumed that deer would locate and consume ash from the most sodiumrich areas, therefore the maximum observed concentration of sodium $(1080 \mathrm{mg} / \mathrm{kg})$ was used to 
represent sodium concentrations in ash.

In the previous exposure estimate, use (and therefore exposure) of the FCAP was assumed to be proportional to the size of the FCAP relative to the size of the home range. In this case, because the FCAP may represent the only available sodium source in the area, it was assumed that deer travel to the site specifically to consume ash and that ash sufficient to satisfy $100 \%$ of the sodium deficit was consumed at the site. Ash consumption was estimated to be $(278 \mathrm{mg}$ $\mathrm{Na} / \mathrm{d}) /(1080 \mathrm{mg} \mathrm{Na} / \mathrm{kg} \mathrm{ash})=0.257 \mathrm{~kg}$ ash $/ \mathrm{d}$. Exposure estimates for consumption of ash by deer to meet sodium requirements are displayed in Table 6. Exposure to three contaminants (arsenic, selenium, and thallium) were from 10 to almost 60 times greater than NOAELs (Table 6), indicating that ash consumption to meet sodium needs may result in adverse effects to whitetailed deer at the FCAP.

\subsubsection{Red-tailed Hawk}

Estimates of contaminant exposure experienced by red-tailed hawk were made using Equation 7 and the following assumptions.

1) body weight $=1.126 \mathrm{~kg}$

2) ash consumption rate $=$ soil consumption rate $=0 \mathrm{~kg} / \mathrm{d}$

3) water consumption rate $=0.064 \mathrm{~L} / \mathrm{d}$

4) food consumption rate $=0.91 \mathrm{~kg} / \mathrm{d}$

5) proportion of mammals in the diet $=0.785$

6) contaminant concentrations measured in small mammals from the FCAP are representative of that in small mammals consumed by red-tailed hawks

7) no other food consumed by red-tailed hawks is contaminated

8) to be conservative, the smallest reported home range was used. Therefore, home range size for red-tailed hawk $=233$ ha

9) use of the FCAP and therefore consumption of food or water from the FCAP is proportional to the size of the FCAP ( $3.6 \mathrm{ha}$ ) red-tailed hawk relative to home range size ( $233 \mathrm{ha}$ ) or 0.0155 ha

Table 7 presents contaminant concentrations and exposure estimates. Contaminant concentrations in ash are in $\mathrm{mg} / \mathrm{kg}$ dry weight while that in food is in $\mathrm{mg} / \mathrm{kg}$ wet weight.

Comparison of the total exposure estimates to NOAELs for each contaminant indicate that no contaminant exceeded NOAELs (Table 7). Because ash consumption was assumed to be negligible, virtually all exposure was obtained through food. 
Table 2. Estimation of contaminant exposure for meadow voles using the FCAP

\begin{tabular}{|c|c|c|c|c|c|c|c|c|c|c|c|}
\hline \multirow[b]{2}{*}{ Contaminant } & \multicolumn{3}{|c|}{$\begin{array}{l}\text { Contaminant Concentration } \\
\text { in Media } \\
\text { (upper } 95 \% \text { confidence interval) }\end{array}$} & \multicolumn{4}{|c|}{$\begin{array}{l}\text { Exposure Estimates } \\
\qquad(\mathrm{mg} / \mathrm{kg} / \mathrm{d})\end{array}$} & \multirow{2}{*}{$\begin{array}{l}\text { NOAELs } \\
\text { for } \\
\text { Meadow } \\
\text { Voles }^{\mathrm{b}} \\
(\mathrm{mg} / \mathrm{kg} / \mathrm{d})\end{array}$} & \multicolumn{3}{|c|}{$\begin{array}{l}\text { Percent of Total Exposure } \\
\text { Attributed to Medium }\end{array}$} \\
\hline & $\begin{array}{l}\text { Water } \\
(\mathrm{mg} / \mathrm{L})\end{array}$ & $\begin{array}{c}\text { Ash } \\
(\mathrm{mg} / \mathrm{kg})\end{array}$ & $\begin{array}{l}\text { Vegetation } \\
(\mathrm{mg} / \mathrm{kg})\end{array}$ & Water & Ash & Food & Total & & Water & Ash & Food \\
\hline Arsenic & 0.01 & 131.00 & 1.77 & 0.00136 & 0.35727 & 0.20114 & 0.55977 & 0.111 & 0.24 & 63.82 & 35.93 \\
\hline Cadmium & $\mathrm{nd}^{\mathrm{c}}$ & nd & 1.23 & 0 & 0 & 0.13977 & 0.13977 & 0.169 & 0 & 0 & 100 \\
\hline Chromium & 0.0088 & 25.10 & 5.30 & 0.0012 & 0.06845 & 0.60227 & 0.67192 & 5425 & 0.18 & 10.19 & 89.63 \\
\hline Lead & 0.0035 & 18.80 & 1.07 & 0.00048 & 0.05127 & 0.12159 & 0.17334 & 15.86 & 0.28 & 29.58 & 70.15 \\
\hline Mercury & nd & 0.705 & 0.06 & 0 & 0.00192 & 0.00682 & 0.00874 & 0.063 & 0 & 21.97 & 78.03 \\
\hline Selenium & nd & 14.80 & 23.61 & 0 & 0.04036 & 2.68295 & 2.72331 & 0.066 & 0 & 1.48 & 98.52 \\
\hline Thallium & nd & 2.21 & 1.00 & 0 & 0.00603 & 0.11364 & 0.11967 & 0.015 & 0 & 5.04 & 94.96 \\
\hline
\end{tabular}

Calculated using Equation 4

b From Opresko et al. 1994

' No data 
Table 3. Estimation of contaminant exposure for red fox using the FCAP

\begin{tabular}{|c|c|c|c|c|c|c|c|c|c|c|}
\hline \multirow[b]{2}{*}{ Contaminant } & \multicolumn{4}{|c|}{$\begin{array}{l}\text { Contaminant Concentration in Media } \\
\text { (upper } 95 \% \text { confidence interval) }\end{array}$} & \multicolumn{5}{|c|}{$\begin{array}{l}\text { Exposure Estimates } \\
(\mathrm{mg} / \mathrm{kg} / \mathrm{d})\end{array}$} & \multirow{2}{*}{$\begin{array}{l}\text { NOAELs } \\
\text { for Red } \\
\text { Fox }^{\mathrm{b}} \\
(\mathrm{mg} / \mathrm{kg} / \mathrm{d})\end{array}$} \\
\hline & $\begin{array}{l}\text { Water } \\
(\mathrm{mg} / \mathrm{L})\end{array}$ & $\begin{array}{c}\text { Ash } \\
(\mathrm{mg} / \mathrm{kg})\end{array}$ & $\begin{array}{l}\text { Mammals } \\
(\mathrm{mg} / \mathrm{kg})\end{array}$ & $\begin{array}{l}\text { Plant } \\
(\mathrm{mg} / \mathrm{kg})\end{array}$ & Water & Soil & $\begin{array}{c}\text { Food } \\
\text { (Mammal) }\end{array}$ & $\begin{array}{l}\text { Food } \\
\text { (Plant) }\end{array}$ & Total & \\
\hline Arsenic & 0.01 & 131.00 & 0.219 & 1.77 & 0.000032 & 0.013755 & 0.000565 & 0.00069 & 0.01504 & 0.024 \\
\hline Cadmium & $\mathrm{nd}^{\mathrm{e}}$ & nd & 0.033 & 1.23 & 0 & 0 & 0.000085 & 0.00048 & 0.00056 & 0.037 \\
\hline Chromium & 0.0088 & 25.10 & 1.528 & 5.30 & 0.000028 & 0.002636 & 0.003942 & 0.002067 & 0.00867 & 1178 \\
\hline Lead & 0.0035 & 18.80 & 2.861 & 1.07 & 0.000011 & 0.001974 & 0.007381 & 0.000417 & 0.00978 & 3.444 \\
\hline Mercury & nd & 0.705 & 0.054 & 0.06 & 0 & 0.000074 & 0.000139 & 0.000023 & 0.00024 & 0.009 \\
\hline Selenium & nd & 14.80 & 3.191 & 23.61 & 0 & 0.001554 & 0.008233 & 0.009208 & 0.01899 & 0.014 \\
\hline Thallium & nd & 2.21 & 0.211 & 1.00 & 0 & 0.000232 & 0.000544 & 0.00039 & 0.00117 & 0.003 \\
\hline
\end{tabular}

Calculated using Equations 14, 15, and 16 .

${ }^{b}$ From Opresko et al. 1994

'No data 
Table 4. Percent of total exposure attributed to medium for red fox using the FCAP

\begin{tabular}{lcccc}
\hline Contaminant & Water & Ash & $\begin{array}{c}\text { Food } \\
\text { (Mammal) }\end{array}$ & $\begin{array}{c}\text { Food } \\
\text { (Plant) }\end{array}$ \\
\hline Arsenic & 0.21 & 91.63 & 3.76 & 4.6 \\
Cadmium & 0 & 0 & 14.25 & 80.29 \\
Chromium & 0.32 & 30.42 & 45.5 & 23.86 \\
Lead & 0.11 & 20.18 & 75.44 & 4.26 \\
Mercury & 0 & 33.03 & 62.17 & 10.43 \\
Selenium & 0 & 8.19 & 43.4 & 48.54 \\
Thallium & 0 & 19.42 & 45.56 & 32.64 \\
\hline
\end{tabular}


Table 5. Estimation of contaminant exposure for white-tailed deer using the FCAP

\begin{tabular}{|c|c|c|c|c|c|c|c|c|c|c|c|}
\hline \multirow[b]{2}{*}{ Contaminant } & \multicolumn{3}{|c|}{$\begin{array}{l}\text { Contaminant Concentration } \\
\text { in Media } \\
\text { (upper 95\% confidence interval) }\end{array}$} & \multicolumn{4}{|c|}{$\begin{array}{c}\text { Exposure Estimates } \\
(\mathrm{mg} / \mathrm{kg} / \mathrm{d})\end{array}$} & \multirow{2}{*}{$\begin{array}{l}\text { NOAELs } \\
\text { for White- } \\
\text { tailed Deer } \\
(\mathrm{mg} / \mathrm{kg} / \mathrm{d})\end{array}$} & \multicolumn{3}{|c|}{$\begin{array}{l}\text { Percent of Total Exposure } \\
\text { Attributed to Medium }\end{array}$} \\
\hline & $\begin{array}{l}\text { Water } \\
(\mathrm{mg} / \mathrm{L})\end{array}$ & $\begin{array}{c}\mathrm{Ash} \\
(\mathrm{mg} / \mathrm{kg})\end{array}$ & $\begin{array}{l}\text { Vegetation } \\
(\mathrm{mg} / \mathrm{kg})\end{array}$ & Water & Ash & Food & Total & & Water & Ash & Food \\
\hline Arsenic & 0.01 & 131.00 & 1.77 & 0.00004 & 0.004923 & 0.003326 & 0.00829 & 0.01 & 0.48 & 59.39 & 40.12 \\
\hline Cadmium & $\mathrm{nd}^{\mathrm{c}}$ & nd & 1.23 & 0 & 0 & 0.002311 & 0.00231 & 0.016 & 0 & 0 & 100 \\
\hline Chromium & 0.0088 & 25.10 & 5.30 & 0.000035 & 0.000943 & 0.009959 & 0.01094 & 511 & 0.32 & 8.62 & 91.04 \\
\hline Lead & 0.0035 & 18.80 & 1.07 & 0.000014 & 0.000707 & 0.002011 & 0.00273 & 1.494 & 0.51 & 25.88 & 73.65 \\
\hline Mercury & nd & 0.705 & 0.06 & 0 & 0.000027 & 0.000113 & 0.00014 & 0.006 & 0 & 18.93 & 80.54 \\
\hline Selenium & nd & 14.80 & 23.61 & 0 & 0.000556 & 0.044366 & 0.04492 & 0.006 & 0 & 1.24 & 98.77 \\
\hline Thallium & nd & 2.21 & 1.00 & 0 & 0.000083 & 0.001879 & 0.00196 & 0.001 & 0 & 4.24 & 95.87 \\
\hline
\end{tabular}

Calculated using Equation 7.

${ }^{b}$ From Opresko et al. 1994

' No data 
Table 6. Estimation of Contaminant Exposure for white-tailed deer consuming ash from the FCAP to meet sodium needs

\begin{tabular}{lcccc}
\hline Contaminant & $\begin{array}{c}\text { Concentration in Ash } \\
(\mathrm{mg} / \mathrm{kg})\end{array}$ & $\begin{array}{c}\text { Exposure Estimates } \\
(\mathrm{mg} / \mathrm{kg} / \mathrm{d})\end{array}$ & $\begin{array}{c}\text { NOAELs } \\
\text { for White-tailed Deer } \\
(\mathrm{mg} / \mathrm{kg} / \mathrm{d})\end{array}$ & $\begin{array}{c}\text { Hazard Quotient } \\
\text { (exposure/NOAEL) }\end{array}$ \\
\hline Arsenic & 131.00 & 0.595876 & 0.01 & 59.6 \\
Cadmium & $\mathrm{nd}^{\mathrm{c}}$ & 0 & 0.016 & 0 \\
Chromium & 25.10 & 0.114172 & 511 & 0.00022 \\
Lead & 18.80 & 0.085515 & 1.494 & 0.06 \\
Mercury & 0.705 & 0.003207 & 0.006 & 0.54 \\
Selenium & 14.80 & 0.06732 & 0.006 & 11.22 \\
Thallium & 2.21 & 0.010053 & 0.001 & 10.05 \\
\hline
\end{tabular}

"Calculated using Equation 4.

${ }^{b}$ From Opresko et al. 1994

'No data 
41

Table 7. Estimation of contaminant exposure for red-tailed hawks using the FCAP

\begin{tabular}{|c|c|c|c|c|c|c|c|c|c|c|c|}
\hline \multirow[b]{2}{*}{ Contaminant } & \multicolumn{3}{|c|}{$\begin{array}{l}\text { Contaminant Concentration } \\
\text { in Media } \\
\text { (upper 95\% confidence interval) }\end{array}$} & \multicolumn{4}{|c|}{$\begin{array}{l}\text { Exposure Estimates } \\
(\mathrm{mg} / \mathrm{kg} / \mathrm{d})\end{array}$} & \multirow{2}{*}{$\begin{array}{l}\text { NOAELs } \\
\text { for Red- } \\
\text { tailed Hawk } \\
(\mathrm{mg} / \mathrm{kg} / \mathrm{d})\end{array}$} & \multicolumn{3}{|c|}{$\begin{array}{l}\text { Percent of Total Exposure } \\
\text { Attributed to Medium }\end{array}$} \\
\hline & $\begin{array}{l}\text { Water } \\
(\mathrm{mg} / \mathrm{L})\end{array}$ & $\begin{array}{c}\text { Ash } \\
(\mathrm{mg} / \mathrm{kg})\end{array}$ & $\begin{array}{c}\text { Mammal } \\
(\mathrm{mg} / \mathrm{kg})\end{array}$ & Water & Ash & $\begin{array}{c}\text { Food } \\
\text { (Mammal) }\end{array}$ & Total & & Water & Ash & $\begin{array}{c}\text { Food } \\
\text { (Mammal) }\end{array}$ \\
\hline Arsenic & 0.01 & 131.00 & 0.219 & 0.000009 & 0 & 0.000257 & 0.00027 & 4.938 & 3.25 & 0 & 95.23 \\
\hline Cadmium & $\mathrm{nd}^{\mathrm{e}}$ & nd & 0.033 & 0 & 0 & 0.000039 & 0.00004 & 1.461 & 0 & 0 & 96.88 \\
\hline Chromium & 0.0088 & 25.10 & 1.528 & 0.000008 & 0 & 0.001794 & 0.0018 & 1.035 & 0.43 & 0 & 99.67 \\
\hline Lead & 0.0035 & 18.80 & 2.861 & 0.000003 & 0 & 0.003359 & 0.00336 & 1.888 & 0.09 & 0 & 99.97 \\
\hline Mercury & nd & 0.705 & 0.054 & 0 & 0 & 0.000063 & 0.00006 & 0.006 & 0 & 0 & 105.67 \\
\hline Selenium & nd & 14.80 & 3.191 & 0 & 0 & 0.003747 & 0.00375 & 0.481 & 0 & 0 & 99.91 \\
\hline Thallium & nd & 2.21 & 0.211 & 0 & 0 & 0.000248 & 0.00025 & nd & 0 & 0 & 99.09 \\
\hline
\end{tabular}

2alculated using Equation 16.

b From Opresko et al. 1994

' No data 


\section{REFERENCES}

Ables, E.D. 1969. Home range studies of red foxes (Vulpes vulpes). J. Mammal. 50: 108120.

Alexander, G.R. 1977. Food of vertebrate predators on trout waters in north central lower Michigan. The Michigan Academician. 10: 181-195.

Althoff, D.P. and G.L. Storm. 1989. Daytime spatial characteristics of cottontail rabbits in central Pennsylvania. J. Mammal. 70: 820-823.

Andersen, D.E. and O.J. Rongstad. 1989. Home-range estimates of red-tailed hawks based on random and systematic relocations. J. Wildl. Manage. 53: 802-807.

Anthony, E. L. P. and T. H. Kunz. 1977. Feeding strategies of the little brown bat, Myotis lucifugus, in southern New Hampshire. Ecology. 58: 775-786.

Arnold, T.W. and E.K. Fritzell. 1987. Food habits of prairie mink during the waterfowl breeding season. Can. J. Zool. 65: 2322-2234.

Arthur, W.J., III and A.W. Alldredge. 1979. Soil ingestion by Mule deer in north central Colorado. J. Range Manage. 32: 67-70.

Arthur, W.J., III and R.J. Gates. 1988. Trace elements intake via soil ingestion in pronghorns and in black-tailed jackrabbits. J. Range Manage. 41: 162-166.

Baes, C.F.,III, R.D. Sharp, A.L. Sjoren, and R.W. Shor. 1984. A review and analysis of parameters for assessing transport of environmentally released radionuclides through agriculture. Oak Ridge National Laboratory, Oak Ridge, TN. ORNL-5786

Barber, H.L. 1984. "Eastern mixed forest." pp. 345-354. In: Halls, L.K., ed. White-tailed Deer: Ecology and Management. Harrisburg, PA: Stackpole Books.

Barrett, G.W., and K.L. Stueck. 1976. Caloric ingestion rate and assimilation efficiency of the short-tailed shrew, Blarina brevicauda. Ohio J. Sci. 76: 25-26.

Batzli, G.O. 1977. Population dynamics of the white-footed mouse in flood plain and upland forests. Am. Midl. Nat. 97: 18-32.

Belwood, J.J. and M.B. Fenton. 1976. Variation in the diet of Myotis lucifugus (Chiroptera: Vespertilionidae). Can. J. Zool. 54: 1674-1678.

Bent, A.C. 1940. Life histories of North American cuckoos, goat suckers, hummingbirds, and their allies. Washington, D.C.: U.S. Government Printing Office; Smithsonian Inst. U.S. Nat. Mus., Bull. 176.

Beyer, W.N. and C. Stafford. 1993. Survey and evaluation of contaminants in earthworms and 
soils from dredged material at confined disposal facilities in the Great Lakes region. Environ. Monit. Assess. 24: 151-165.

Beyer, W.N., E. Conner, and S. Gerould. 1994. Estimates of soil ingestion by wildlife. J. Wildl. Manage. 58: 375-382.

Bleavins, M.R. and R.J. Aulerich. 1981. Feed consumption and food passage time in mink (Mustela vison) and European ferrets (Mustela putorius furo). Lab. Anim. Sci. 31: 268-269.

Brooks, R.P and W.J. Davis. 1987. Habitat selection by breeding belted kingfishers (Ceryle alcyon). Am. Midl. Nat. 117: 63-70.

Buckner, C.H. 1964. Metabolism, food capacity, and feeding behavior in four species of shrews. Can. J. Zool. 42: 259-279.

Buckner, C.H. 1966. Populations and ecological relationships of shrews in Tamarack bogs of southeastern Manitoba. J. Mammal. 47: 181-194.

Burgess, S.A. and J.R. Bider. 1980. Effects of stream habitat improvements on invertebrates, trout populations, and mink activity. J. Wildl. Manage. 44: 871-880.

Burt, W.H. and R.P. Grossenheider. 1976. A field guide to the mammals of America north of Mexico. Third Edition. Houghton Mifflin Co., Boston

Calder, W.A. and E.J. Braun. 1983. Scaling of osmotic regulation in mammals and birds. Am. J. Physiol. 224: Rr601-R606.

Camner, P., T.W. Clarkson, and G.F. Nordberg. 1979. "Routes of exposure, dose and metabolism of metals." pp 65-97. In: Friberg, L., G.F. Nordberg, and V.B. Vouk, eds. Handbook on the toxicology of metals. Amsterdam, The Netherlands: Elsevier/North-Holland Biomedical Press.

Chapman, J.A., J.G. Hockman, and M.M. Ojeda C. 1980. Sylvilagus floridanus. Mammal. Species. No. 136, pp. 1-8.

Chew, R.M. 1951. The water exchanges of some small mammals. Ecol. Monogr. 1: 215-224.

Collazo, J.A. 1985. Food habits of nesting Great Blue herons at Heyburn State Park, Idaho. Northwest Science. 59: 144-146.

Connors, J.I. and P.D. Doerr. 1982. Woodcock use of agricultural fields in coastal North Carolina. In: Dwyer, T.J., Storm, G.W., tech. coords. Woodcock ecology and management. U.S. Fish Wildl. Sevr., Wildl. Res. Rep. 14: 139-147.

Coon, R.A., B.K. Williams, J.S. Lindzey, et al. 1982. Examination of woodcock nest sites in central Pennsylvania. U.S. Fish Wildl. Serv., Wildl. Res. Rep. 14: 55-62. 
Craighead, J.J., and F.C. Craighead, Jr. 1969. Hawks, owls, and wildlife. Dover Publ., Inc. New York. $443 \mathrm{pp}$.

Dalke, P.D. and P.R. Sime. 1941. Food habits of the eastern and New England cottontails. J. Wildl. Manage. 5: 216-228.

Dark, J., I. Zucker, and G.N. Wade. 1983. Photoperiodic regulation of body mass, food intake, and reproduction in meadow voles. Am. J. Physiol. 245: R334-R338.

Davis, W.J. 1982. Territory size in Megaceryle alcyon along a stream habitat. $A u k$. 99: 353362.

DeGraaf, R.M. and D.D. Rudis. 1986. New England Wildlife: Habitat, Natural History and Distribution. USDA Forest Serv. GTR NE-108. 491 p.

Dunning, J.B. 1984. Body weights of 686 species of North American birds. West. Bird Banding Assoc. Monogr. No. 1. Eldon Publ. Co. Cave Crk, AZ. 38 pp.

Eadie, W.R. 1952. Shrew predation and vole populations on a localized area. J. Mammal. 33: 185-189.

Eberhardt, L., T.J. Peterle, and R. Schofield. 1963. Problems in a rabbit population study. Wildl. Monogr. No. 10: 6-7.

Eiserer, L.A. 1980. Effects of grass length and mowing on foraging behavior of the American robin (Turdus migratorius). Auk. 97: 576-580.

EPA (U. S. Environmental Protection Agency). 1992. Dermal exposure assessment: principles and applications. Office of Health and Environmental Assessment, Washington, D.C. EPA/600/8-91/011B.

EPA (U. S. Environmental Protection Agency). 1993a. Wildlife exposure factors handbook. Volume I. Office of Research and Development, Washington, DC. EPA/600/R-93/187a.

EPA (U. S. Environmental Protection Agency). 1993b. Wildlife criteria portions of the proposed water quality guidance for the Great Lakes system. EPA/822/R-93/006. Office of Science and Technology, Washington, D.C.

Fenton, M.B. and G.P. Bell. 1979. Ecolocation and feeding behavior in four species of Myotis (Chiroptera). Can. J. Zool. 57: 1271-1277.

Fenton, M.B. and R.M.R. Barclay. 1980. Myotis lucifugus. Mammal. Species. 142: 1-8.

Garten, C.T., Jr. 1980. Ingestion of soil by Hispid cotton rats, white-footed mice, and eastern chipmunks. J. Mammal. 6: 136-137.

George, S.B., J.R. Choate, and H.H. Genoways. 1986. Blarina brevicauda. Mammal. species. 
261: $1-9$.

Gerell, R. 1970. Home ranges and movements of the mink Mustela vison Schreber in southern Sweden. Oikos 21: 160-173.

Getz, L.L. 1961. Factors influencing the local distribution of Microtus and Synaotomys in southern Michigan. Ecology. 42: 110-119.

Getz, L.L. 1989. A 14-year study of Blarina brevicauda populations in east-central Illinois. J. Mammal. 70: 58-66.

Gish, C.D. and D.L. Hughes. 1982. Residues of DDT, dieldrin, and heptachlor in earthworms two years following application. U.S. Fish and Wildlife Service, Spec. Sci. Rep., Wildlife. No. 241.

Gould, E.D. 1955. The feeding efficiency of insectivorous bats. J. Mammal. 36: 399-407

Green, D.A., and J.S. Millar. 1987. Changes in gut dimensions and capacity of Peromyscus maniculatus relative to diet quality and energy needs. Can. J. Zool. 65: 2159-2162.

Hamilton, W.J.,Jr. 1940. The summer food of minks and raccoons on the Montezuma Marsh, New York. J. Wildl. Manage. 4: 80-84.

Hazelton, P.K., R. J. Robel, and A.D. Dayton. 1984. Preferences and influence of paired food items on energy intake of American robins and gray catbirds. J. Wildl. Manage. 48: 198202.

Heppner, F. 1965. Sensory mechanisms and environmental clues used by the American Robin in locating earthworms. Condor. 67:247-256.

Hoffman, R.D. 1978. The diets of herons and egrets in southwestern Lake Erie. Natl. Audubon Soc. Res. Ref. 7: 365-369.

Hudgins, J.E., G.L. Storm, and J.S. Wakeley. 1985. Local movements and diurnal-habitat selection by male American woodcock in Pennsylvania. J. Wildl. Manage. 49: 615-619.

Janes, S.W. 1984. Influences of territory composition and interspecific competition on red-tailed hawk reproductive success. Ecology. 65: 862-870.

Johnsgard, P.A. 1988. North American Owls: biology and natural history. Smithsonian Institution Press, Washington.

Keen, R. and H.B. Hitchcock. 1980. Survival and longevity of the little brown bat (Myotis lucifugus) in southeastern Ontario. J. Mammal. 61: 1-7.

Knight, R.L. and R.E. Jackman. 1984. Food-niche relationship between great horned owls and common barn-owls in eastern Washington. Auk. 101: 175-179. 
Korschgen, L.J. 1958. December food habits of mink in Missouri. J. Mammal. 39: 521-527.

Korschgen, L.J. 1967. "Feeding habits and foods." pp. 137-198. In: The Wild Turkey and Its Management.

Krohn, W.B. 1970. Woodcock feeding habits as related to summer field usage in central Maine. J. Wildl. Manage. 34: 769-775.

Kushlan, J.A. 1978. Feeding ecology of wading birds. Wading Birds. National Audubon Society. p. 249-297.

Landrum, C.L., T.L. Ashwood, and D.K. Cox. 1993. Belted kingfishers as ecological monitors of contaminations: a review. ORNL/M-2533. Oak Ridge National Laboratory, Oak Ridge, TN.

LaVal, R.K, R.L. Clawson, M.L. LaVal, and W. Caire. 1977. Foraging behavior and nocturnal activity patterns of Missouri bats, with emphasis on the endangered species Myotis grisescens and Myotis sodalis. J. Mammal. 58: 592-599.

Lackey, J.A., D.G. Huckaby, and B.G. Ormiston. 1985. Peromyscus leucopus. Mammal. Species 247: 1-10.

Lindroth, R.L. and G.O. Batzli. 1984. Food habits of the meadow vole (Microtus pennsylvanicus) in bluegrass and prairie habitats. J. Mammal. 65: 600-606.

Linscombe, G., N. Kinler, R.J. Aulerich. 1982. "Mink." pp. 329-643. In: Chapman, J.A., A. Feldhammer, eds. Wild mammals of North America. Baltimore, MD: John Hopkins University Press.

Marchinton, R.L. and D.H. Hirth. 1984. "Behavior." pp. 129-168. In: Halls, L.K., ed. White-tailed Deer: Ecology and Management. Harrisburg, PA: Stackpole Books.

Mautz, W.W., H. Silver, J.B. Hayes, and W.E. Urban. 1976. Digestibility and related nutritional data for seven northern deer browse species. J. Wildl. Manage. 40: 630-638.

Menzie, C.A., D.E. Burmaster, J.S. Freshman, and C.A. Callahan. 1992. Assessment of methods for estimating ecological risk in the terrestrial component: a case study at the Baird and McGuire Superfund site in Holbrook, Massachusetts. Environ. Toxicol. Chem. 11: 245260.

Miller, D.H. and L.L. Getz. 1977. Factors influencing local distribution and species diversity of forest small mammals in New England. Can. J. Zool. 55: 806-814.

Miller, D.L. and M.K. Causey. 1985. Food preferences of American woodcock wintering in Alabama. J. Wildl. Manage. 49: 492-496.

Mitchell, J.L. 1961. Mink movements and population on a Montana river. J. Wildl. Manage. 
25: $48-54$.

Nagy, K.A. 1987. Field metabolic rate and food requirement scaling in mammals and birds. Eco. Monogr. 57: 111-128.

National Geographic Society. 1987. Field Guide to the Birds of North America. 2nd Edition.

Newell, A.J., D.W. Johnson, and L.K. Allen. 1987. Niagara River biota contamination project: fish flesh criteria for piscivorous wildlife. Division of Fish and Wildlife, Bureau of Environmental Protection. Technical report 87-3.

Opresko, D.M., B.E. Sample, and G.W. Suter, III. 1994. Toxicological benchmarks for wildlife. ES/ER/TM-6/R1. Oak Ridge National Laboratory, Oak Ridge, TN.

Orians, G.H. and F. Kuhlman. 1956. Red-tailed hawk and horned owl populations in Wisconsin. Condor 58: 371-385.

Ostfeld, R.S., S.R. Pugh, J.O. Seamon, and R.H. Tamarin. 1988. Space use and reproductive success in a population of meadow voles. J. Animal Ecology. 57: 385-394.

Oswald, C., P.Fonken, D. Atkinson, and M. Palladino. 1993. Lactational water balance and recycling in white-footed mice, red-backed voles, and gerbils. J. Mammal. 74: 963-970.

Pitts, T.D. 1984. Description of American robin territories in northwest Tennessee. Migrant. 55: $1-6$.

Pletscher, D.H. 1987. Nutrient budgets for white-tailed deer in New England with special reference to sodium. J. Mammal. 68:330-336.

Pough, R.H. 1949. Audubon bird guide: Eastern land birds. Doubleday and Co., Inc., New York. 312 p.

Pough, R.H. 1951. Audubon water bird guide. Doubleday and Co., Inc., New York.

Preston, C.R. 1990. Distribution of raptor foraging in relation to prey biomass and habitat structure. Condor 92: 107-112.

Reich, L.M. 1981. Microtus pennsylvanicus. Mammal. Species. 159: 1-8.

Risk Assessment Forum. 1992. Framework for ecological risk assessment. EPA/630/R-92/001. U.S. Environmental Protection Agency, Washington, D.C.

Robbins, C.T. 1993. Wildlife feeding and nutrition. Academic Press, San Diego, CA.

Sargeant, A.B. 1972. Red fox spatial characteristics in relation to waterfowl predation. $J$. Wildl. Manage. 36: 225-236. 
Sargeant, A.B. 1978. Red fox prey demands and implications to prairie duck production. $J$. Wildl. Manage. 42(3): 520-527.

Schlessinger, W.H. and G.L. Potter. 1974. Lead, copper, and cadmium concentrations in small mammals in the Hubbard Brook experimental forest. Oikos. 25: 148-152.

Sealander, J.A. 1943. Winter food habits of mink in southern Michigan. J. Wildl. Manage. 7: $411-417$.

Sheldon, W.G. 1971. The book of the American woodcock. University of Massachusetts Press.

Short, H.L. and R.J. Cooper. 1985. Habitat suitability index models: Great Blue heron. Biological report 82(10.99). Fish and Wildlife Service, U.S. Department of the Interior.

Skorupa, J.P. and R.L. Hothem. 1985. Consumption of commercially-grown grapes by American robins: a field evaluation of laboratory estimates. J. Field Ornithol. 56: 369-378.

Smith W.P. 1991. Odocoileus virginianus. Mammal. Species. 388: 1-13.

Sperry, C.C. 1940. Food habits of a group of shorebirds: woodcock, snipe, knot, and dowitcher. Wildl. Res. Bull. 1. U.S. Government Printing Office, Washington D.C.

Storm, G.L., R.D. Andrews, R. L. Phillips, R.A. Bishop, D.B. Siniff, and J.R. Tester. 1976. Morphology, reproduction, dispersal, and mortality of midwestern red fox populations. Wildl. Monogr.

Stribling, H.L. and P.D. Doerr. 1985. Nocturnal use of fields by American woodcock. J. Wildl. Manage. 49: 485-491.

Suter, G.W., II. 1989. "Ecological endpoints." pp. 2-1 to 2-28. In: Warren-Hicks, W., R. Parkhurst, and S.S. Baker, Jr.(eds.). Ecological assessment of hazardous waste sites: a field and laboratory reference document. EPA/600/3-89/013. U.S. Environmental Protection Agency, Washington, D.C.

Suter, G.W., II., B. E. Sample, D. S. Jones, and T. L. Ashwood. 1994. Approach and strategy for performing ecological risk assessments on the Oak Ridge Reservation: 1994 revision. ES/ER/TM-33/R1. Oak Ridge National Laboratory, Oak Ridge, TN.

Talmage, S.S. and B.T. Walton. 1993. Food chain transfer and potential renal toxicity to small mammals at a contaminated terrestrial field site. Ecotoxicology. 2: 243-256

Thornton, I. and P. Abrahams. 1983. Soil ingestion - a major pathway of heavy metals into livestock grazing contaminated land. Sci. Tot. Environ. 28: 27-294.

Torgerson, O. and W.R. Porath. 1984. "Midwest oak/hickory forest." pp. 411-426. In: Halls, L.K., ed. White-Tailed Deer: Ecology and Management. Stackpole Books, Harrisburg, PA. 
Travis, C.C. and A.D. Arms. 1988. Bioconcentration of organics in beef, milk, and vegetation. Environ. Sci. Technol. 22: 271-274.

van Zyll de Jong, C.G. 1983. Handbook of Canadian Mammals. National Museums of Canada. pp. 210.

Vogtsberger, L.M. and G.W. Barrett. 1973. Bioenergetics of captive red foxes. J. Wildl. Manage. 37: 495-500.

Wallace, J.G. 1948. The barn owl in Michigan, its distribution, natural history and food habits. Mich. State Col. Agric. Exp. Tech. Bull. No. 208.

Watters, R.L., D.N. Edgington, T.E. Hakonson, W.C. Hanson, M.H. Smith, F.W. Whicker, and R.E. Wildung. 1980. "Synthesis of the Research Literature." In: Transuranic elements in the environment. U. S. Department of Energy.

Weeks, H.P., Jr. 1978. Characteristics of mineral licks and behavior of visiting white-tailed deer in southern Indiana. Am. Midl. Nat. 100: 384.395.

Wheelwright, N.T. 1986. The diet of American robins: an analysis of U.S. Biological Survey records. Auk. 103: 710-725.

Whitaker, J.O., Jr. and M.G. Ferraro. 1963. Summer food of 220 short-tailed shrews from Ithaca, New York. J. Mammal. 44: 419.

Whitaker, J.O., Jr. 1966. Food of Mus musculus, Peromyscus maniculatus bairdii, and Peromyscus leucopus in Vigo County, Indiana. J. Mammal. 47: 473-486.

Wolff, J.O., R.D. Dueser, and K. S. Berry. 1985. Food habits of sympatric Peromyscus leucopus and Peromyscus maniculatus. J. Mammal. 66: 795-798.

Wolff, J.O. 1985. The effects of density, food, and interspecific interference on home range size in Peromyscus leucopus and Peromyscus maniculatus. Can. J. Zool. 63:2657-2662.

Young, H. 1951. Territorial behavior in the eastern robin. Proc. Linnaean Soc. NY. 58-62: 1-37.

Zim, H.S., A.C. Martin, and A.L. Nelson. 1951. American wildlife and plants a guide to wildlife food habits. Dover Publications, Inc. New York. 
1. J. Archer

2. L. W. Barnthouse

3. L. Baron

4. B. G. Blaylock

5. R. R. Bonczek

6. M. Clauberg

7. J. Dee

8. J. R. Duncan

9. M. Ferré

10. D. Gonzales

11. R. N. Hull

12. D. S. Jones

13. R. C. Kramel

14. S. Lampkins

15. M. Leslie

16. R. Mathis

17-9. D. M. Matteo

20. C. W. McGinn

21. D. Mentzer

22. P. D. Miller

23. D. B. Miller
24. B. D. Nourse

25-6. P. T. Owen

27. S. Pack

28. S. T. Purucker

29. Sue Reith

30. B. E. Sample

31. D. M. Steinhauff

32. G. Stephens

33. G. W. Suter

34. Andrea Temeshy

35. C. C. Travis

36. C. J. E. Welch

37. R. K. White

38. Don Wilkes

39. E. Will

40. Central Research Library

41-2. ER Document Management Center

43. Laboratory Records

44. ORNL Patent Section

45. D. M. Opresko

46. M. E. Will

47. Office of Assistant Manager for Energy Research and Development, DOE Oak Ridge Field Office, P.O. Box 2001, Oak Ridge, TN 37831-8600.

48. Office of Scientific and Technical Information (OSTI), P.O. Box 62, Oak Ridge, TN, 37831. 